\title{
A DEGREE PROBLEM FOR TWO ALGEBRAIC NUMBERS AND THEIR SUM
}

\author{
Paulius Drungilas, Artūras Dubickas, and Chris Smyth
}

\begin{abstract}
For all but one positive integer triplet $(a, b, c)$ with $a \leqslant b \leqslant c$ and $b \leqslant 6$, we decide whether there are algebraic numbers $\alpha, \beta$ and $\gamma$ of degrees $a, b$ and $c$, respectively, such that $\alpha+\beta+\gamma=0$. The undecided case $(6,6,8)$ will be included in another paper. These results imply, for example, that the sum of two algebraic numbers of degree 6 can be of degree 15 but cannot be of degree 10. We also show that if a positive integer triplet $(a, b, c)$ satisfies a certain triangle-like inequality with respect to every prime number then there exist algebraic numbers $\alpha, \beta, \gamma$ of degrees $a, b, c$ such that $\alpha+\beta+\gamma=0$. We also solve a similar problem for all $(a, b, c)$ with $a \leqslant b \leqslant c$ and $b \leqslant 6$ by finding for which $a, b, c$ there exist number fields of degrees $a$ and $b$ such that their compositum has degree $c$. Further, we have some results on the multiplicative version of the first problem, asking for which triplets $(a, b, c)$ there are algebraic numbers $\alpha, \beta$ and $\gamma$ of degrees $a, b$ and $c$, respectively, such that $\alpha \beta \gamma=1$.
\end{abstract}

2010 Mathematics Subject Classification: 11R04, 11R32.

Key words: algebraic number, sum-feasible, $a b c$ degree problem.

\section{Introduction and results}

The purpose of this paper is to propose the following problem:

Find all possible triplets $(a, b, c) \in \mathbb{N}^{3}$ for which there exist three algebraic numbers $\alpha, \beta, \gamma$, with degrees $a, b, c$ (over $\mathbb{Q}$ ), respectively, such that

$$
\alpha+\beta+\gamma=0 .
$$

This is our abc degree problem for algebraic numbers. When such $\alpha, \beta, \gamma$ exist, we say that the triplet $(a, b, c)$ is sum-feasible. It seems that this $a b c$ degree problem for sums of algebraic numbers is unrelated to the famous abc conjecture for integers proposed by Oesterlé and Masser in 1985 .

Even for small values of $a, b$ and $c$ it is sometimes very difficult to decide whether the triplet $(a, b, c)$ is sum-feasible. See, for instance, 
the proof of Theorem 38, where we establish that $(6,6,10)$ is not sumfeasible. With the methods used here we were, however, unable to settle our $a b c$ degree problem in the case $(6,6,8)$. This case has now been shown elsewhere to not be sum-feasible - see [6].

We propose a similar problem for the compositum of fields by saying that a triplet $(a, b, c) \in \mathbb{N}^{3}$ is compositum-feasible if there are number fields $K$ and $L$ of degrees $a$ and $b$, respectively, over the field of rationals $\mathbb{Q}$ such that the degree of their compositum $K L$ is $c$. For example, the triplet $(2,2,4)$ is compositum-feasible $(K=\mathbb{Q}(\sqrt{2}), L=\mathbb{Q}(\sqrt{3})$, $K L=\mathbb{Q}(\sqrt{2}, \sqrt{3}))$, whereas the triplet $(2,2,5)$ is not compositumfeasible, since $[K L: \mathbb{Q}]$ cannot exceed $[K: \mathbb{Q}] \cdot[L: \mathbb{Q}]$.

Similarly, we say that a triplet $(a, b, c) \in \mathbb{N}^{3}$ is product-feasible if there are algebraic numbers $\alpha, \beta$ and $\gamma$ of degrees (over $\mathbb{Q}$ ) $a, b$ and $c$, respectively, such that $\alpha \beta \gamma=1$.

Note that if a triplet $(a, b, c), a \leqslant b \leqslant c$, is sum-feasible, compositumfeasible or product-feasible then $c \leqslant a b$. If $(a, b, c), a \leqslant b \leqslant c$, is compositum-feasible then $a \mid c$ and $b \mid c$. These are obvious necessary conditions. In Section 2 we give another simple necessary condition for a triplet to be sum-feasible, compositum-feasible or product-feasible (see Lemma 14).

These three problems are related.

Proposition 1. If the triplet $(a, b, c) \in \mathbb{N}^{3}$ is compositum-feasible then it is also sum-feasible and product-feasible.

Proof: Suppose that $K$ and $L$ are number fields. Then, by the primitive element theorem, $K=\mathbb{Q}(\alpha)$ and $L=\mathbb{Q}(\beta)$ for some $\alpha \in K$ and $\beta \in L$. Furthermore, the compositum $K L=\mathbb{Q}(\alpha, \beta)$ can be expressed as $K L=$ $\mathbb{Q}(\alpha+t \beta)$ and also as $K L=\mathbb{Q}(\alpha(t+\beta))$ for all but finitely many rational numbers $t$. See the proof of Theorem 4.6 in [16] for the case $\alpha+t \beta$. The proof for $\alpha(t+\beta)$ is the same. Indeed, consider the field $K_{t}=\mathbb{Q}(\alpha(t+\beta))$. Since $\mathbb{Q} \subseteq K_{t} \subseteq \mathbb{Q}(\alpha, \beta)$, there are two distinct rational numbers $t$ and $t^{\prime}$ for which $K_{t}=K_{t^{\prime}}$. Assume without loss of generality that $\alpha\left(t^{\prime}+\beta\right) \neq 0$. Then, as the quotient of $\alpha(t+\beta)$ and $\alpha\left(t^{\prime}+\beta\right)$ belongs to $K_{t}$, we obtain $\left(t-t^{\prime}\right) /\left(t^{\prime}+\beta\right)=(t+\beta) /\left(t^{\prime}+\beta\right)-1 \in K_{t}$. Thus $\beta \in K_{t}$. This implies $\alpha \in K_{t}$, so that $K_{t}=\mathbb{Q}(\alpha, \beta)$.

Since $[K: \mathbb{Q}]=a,[L: \mathbb{Q}]=b,[K L: \mathbb{Q}]=c$, choosing an appropriate $t \in \mathbb{Q}$, in the additive case we see that the degrees of $\alpha, t \beta$ and $-\alpha-t \beta$ are $a, b, c$, respectively. In the multiplicative case, the degrees of $\alpha, t+\beta$ and $\alpha^{-1}(t+\beta)^{-1}$ are $a, b, c$. 
The converse of Proposition 1 is false in general. Clearly, if the triplet $(a, b, c)$ is sum-feasible (resp. product-feasible) then for any permutation $\left\{a^{\prime}, b^{\prime}, c^{\prime}\right\}$ of $\{a, b, c\}$ the triplet $\left(a^{\prime}, b^{\prime}, c^{\prime}\right)$ is also sum-feasible (resp. product-feasible). However, the compositum problem is not symmetric with respect to $a, b, c$. The triplet $(n, n, 1), n>1$, is not compositum-feasible, since the degree of the compositum of two number fields of degree $n$ is divisible by $n$. Meanwhile $(n, n, 1)$ is sum-feasible and product-feasible: for $\alpha=\sqrt[n]{2}, \beta=-\alpha, \gamma=0$ we have $\alpha+\beta+\gamma=0$, whereas $\alpha^{\prime}=\sqrt[n]{2}, \beta^{\prime}=\alpha^{\prime-1}, \gamma^{\prime}=1$ gives $\alpha^{\prime} \beta^{\prime} \gamma^{\prime}=1$. The less trivial example $(4,4,6)$ (which is sum-feasible and product-feasible but not compositum-feasible) follows from Proposition 29 (ii) (Section 3). The reason for not being compositum-feasible is that 4 does not divide 6 . We do not know of any example $(a, b, c) \in \mathbb{N}^{3}$ satisfying $a|c, b| c$ which is sum-feasible (or product-feasible) but is not compositum-feasible.

We have found little in the literature directly related to our problem apart from the 'generic' case $(a, b, a b)$ which has long been known to be compositum-feasible (and hence sum-feasible and product-feasible) - see Proposition 19 below. In particular, one result, due to Isaacs [11] who generalized an earlier result of Kaplansky [15, p. 71], implies that if $\alpha$ has degree $a$ (over $\mathbb{Q}$ ), $\beta$ has degree $b$ and $\operatorname{gcd}(a, b)=1$ then $\alpha+\beta$ has degree $a b$. Let us state this result in the following symmetric form.

Proposition $2([\mathbf{1 1}])$. If the triplet $(a, b, c) \in \mathbb{N}^{3}$ is sum-feasible and two particular numbers from the list $a, b, c$ are coprime then the third number is the product of these two.

See also [2], [7] and [8], where some conditions for the degree of $\alpha+\beta$ to be 'maximal possible' $\operatorname{deg}(\alpha) \cdot \operatorname{deg}(\beta)$ are given without assumption that $\operatorname{deg}(\alpha)$ and $\operatorname{deg}(\beta)$ are coprime. (Throughout, we denote by $\operatorname{deg}(\alpha)$ the degree of an algebraic number $\alpha$ over $\mathbb{Q}$.) In particular, it is remarked in [2, p. 261] that the proof of Isaac's result quoted above shows that if $(a, b, a b)$ is compositum-feasible then it is sum-feasible (i.e., a special case of Proposition 1).

We conjecture that

Conjecture 3. If the triplet $(a, b, c) \in \mathbb{N}^{3}$ is sum-feasible then it is also product-feasible.

The converse of Conjecture 3 is false. The triplet $(2,3,3)$ is not sumfeasible, by Proposition 2 . Hence $(2,3,3)$ is not compositum-feasible either. However, $(2,3,3)$ is product-feasible. For example, the numbers

$$
\alpha=(-1-i \sqrt{3}) / 4, \quad \beta=\sqrt[3]{2}, \quad \gamma=(-1+i \sqrt{3}) / \sqrt[3]{2}
$$

have product 1 and degrees $2,3,3$, respectively. 
Conjecture 4. If the triplets $(a, b, c),\left(a^{\prime}, b^{\prime}, c^{\prime}\right) \in \mathbb{N}^{3}$ are sum-feasible (resp. product-feasible, compositum-feasible) then the triplet $\left(a a^{\prime}, b b^{\prime}, c c^{\prime}\right)$ is also sum-feasible (resp. product-feasible, compositum-feasible).

Some partial cases of Conjecture 4 are given in Lemma 26, Corollary 27, Proposition 28 and Proposition 32.

The main result of this paper is the following.

Theorem 5. All the triplets $(a, b, c)$ of positive integers with $a \leqslant b \leqslant$ $c, b \leqslant 6$ that are sum-feasible are given in Table 1 , with one possible exception $(6,6,8)$. Every such triplet is also compositum-feasible, except for $(4,4,6),(4,6,6),(6,6,8),(6,6,9)$ and $(6,6,15)$.

TABLE 1 . Triplets $(a, b, c), a \leqslant b \leqslant c, b \leqslant 6$, that are sumfeasible. (Because of $[\mathbf{6}$, Theorem 1$]$, the case $(6,6,8)$ does not appear.)

\begin{tabular}{|c|c|c|c|c|c|c|}
\hline $\mathbf{b} \backslash \mathbf{a}$ & 1 & 2 & 3 & 4 & 5 & 6 \\
\hline 1 & 1 & & & & & \\
\hline 2 & 2 & 2,4 & & & & \\
\hline 3 & 3 & 6 & $3,6,9$ & & & \\
\hline 4 & 4 & 4,8 & 12 & $\begin{array}{l}4,6,8 \\
12,16\end{array}$ & & \\
\hline 5 & 5 & 10 & 15 & 20 & $\begin{array}{c}5,10 \\
20,25\end{array}$ & \\
\hline 6 & 6 & 6,12 & $6,12,18$ & $6,12,24$ & 30 & $\begin{array}{c}6,9,12, \\
15,18,24, \\
30,36\end{array}$ \\
\hline
\end{tabular}

By our observation above (Proposition 1 ), if the triplet $(a, b, c)$ is not sum-feasible then it is not compositum-feasible. For example, there are exactly five triplets $(a, b, c), a \leqslant b \leqslant c$, with $a=b=4$ that are sumfeasible, namely $(4,4,4),(4,4,6),(4,4,8),(4,4,12)$ and $(4,4,16)$. However, since 6 is not a multiple of 4 , the triplet $(4,4,6)$ is not compositumfeasible. Taking $K=\mathbb{Q}(\sqrt{2}, \sqrt{3})$ and $L$, say, $\mathbb{Q}(\sqrt{2}, \sqrt{3}), \mathbb{Q}(\sqrt{2}, \sqrt{5})$, 
$\mathbb{Q}(\sqrt{5}, \sqrt{7})$ we see that the triplets $(4,4,4),(4,4,8),(4,4,16)$ are compositum-feasible. Further, taking any quartic algebraic number $\alpha$ such that the Galois group of $\mathbb{Q}(\alpha)$ over $\mathbb{Q}$ is the full symmetric group $S_{4}$ and its conjugate $\alpha^{\prime} \neq \alpha$, we see that $[\mathbb{Q}(\alpha): \mathbb{Q}]=\left[\mathbb{Q}\left(\alpha^{\prime}\right): \mathbb{Q}\right]=4$ and $\left[\mathbb{Q}\left(\alpha, \alpha^{\prime}\right): \mathbb{Q}\right]=12$. This shows that the triplet $(4,4,12)$ is also compositum-feasible.

Let $p$ be a prime number. For a positive integer $n$ we define the nonnegative integer $\operatorname{ord}_{p}(n)$ by

$$
p^{\operatorname{ord}_{p}(n)} \mid n \text { and } \quad p^{\operatorname{ord}_{p}(n)+1} \nmid n .
$$

We say that a triplet $(a, b, c)$ satisfies the exponent triangle inequality with respect to a prime number $p$ if

$$
\begin{aligned}
\operatorname{ord}_{p}(a)+\operatorname{ord}_{p}(b) & \geqslant \operatorname{ord}_{p}(c), \quad \operatorname{ord}_{p}(b)+\operatorname{ord}_{p}(c) \geqslant \operatorname{ord}_{p}(a) \quad \text { and } \\
& \operatorname{ord}_{p}(a)+\operatorname{ord}_{p}(c) \geqslant \operatorname{ord}_{p}(b) .
\end{aligned}
$$

For example, the triplet $(6,6,10)$ satisfies the exponent triangle inequality with respect to every prime number $p$ except for $p=5$.

Theorem 6. If a triplet of positive integers $(a, b, c)$ satisfies the exponent triangle inequality with respect to every prime number then the triplet $(a, b, c)$ is sum-feasible and product-feasible.

The exponent triangle inequality condition in Theorem 6 is not necessary. For instance, the triplet $(3,3,6)$ is sum-feasible and productfeasible (e.g., if $\alpha$ and $\alpha^{\prime}$ are two distinct roots of the polynomial $x^{3}-x-1$ then the degrees of $\alpha$ and $\alpha^{\prime-1}$ are 3, the degree of $\alpha^{\prime} / \alpha$ is 6 and $\left.\alpha \cdot \alpha^{\prime-1} \cdot\left(\alpha^{\prime} / \alpha\right)=1\right)$, while the exponent triangle inequality with respect to the prime number 2 is not satisfied. In fact, one can be 'very far' from the exponent triangle inequality. By Proposition 29 (i) combined with Proposition 1, the triplet $(a, b, c)=\left(2^{m}+1,2^{m}+1,2^{m}\left(2^{m}+1\right)\right)$ is sum-feasible, whereas

$$
\operatorname{ord}_{2}(c)-\operatorname{ord}_{2}(b)-\operatorname{ord}_{2}(a)=m
$$

can be arbitrarily large.

We remark that the condition of Theorem 6 is not sufficient for a triplet to be compositum-feasible. For example, the triplet $(6,10,15)$ satisfies the exponent triangle inequality with respect to every prime number. However, it is not compositum-feasible, because the compositum of two extensions of $\mathbb{Q}$ of degrees 6 and 10 has degree divisible by $\operatorname{lcm}(6,10)=30$. 
More generally, an extra condition for a triplet $(a, b, c) \in \mathbb{N}^{3}$ to be compositum-feasible can be written as

$$
\max \left\{\operatorname{ord}_{p}(a), \operatorname{ord}_{p}(b)\right\} \leqslant \operatorname{ord}_{p}(c)
$$

for every prime number $p$. This necessary condition becomes sufficient for triplets $(a, b, c)$ satisfying the exponent triangle inequality with respect to any prime number. This result can readily be written in the following form.

Theorem 7. If a triplet of positive integers $(a, b, c)$ satisfies

$$
\max \left\{\operatorname{ord}_{p}(a), \operatorname{ord}_{p}(b)\right\} \leqslant \operatorname{ord}_{p}(c) \leqslant \operatorname{ord}_{p}(a)+\operatorname{ord}_{p}(b)
$$

for every prime number $p$ then the triplet $(a, b, c)$ is compositum-feasible.

Let $(a, b, c)$ be any triplet of positive integers. It is easy to see that the triplet $\left(a(a b c)^{n}, b(a b c)^{n}, c(a b c)^{n+1}\right)$ satisfies (1) for all primes $p$ provided that $n$ is large enough. Therefore Theorem 7 implies that the triplet $\left(a(a b c)^{n}, b(a b c)^{n}, c(a b c)^{n+1}\right)$ is compositum-feasible (and hence sum-feasible and product-feasible) for each sufficiently large $n \in \mathbb{N}$.

Note that for $p$ a prime number and $t \in \mathbb{N}$ the triplet $(p, t, t)$ is sumfeasible if and only if $p \mid t$. The necessity follows from Proposition 2 , the sufficiency from the example $\alpha=-2 \cdot 2^{1 / p}, \beta=2^{1 / p}+3^{p / t}, \gamma=2^{1 / p}-3^{p / t}$, where $t$ is a positive integer divisible by $p$. In particular, for $p=2$, the triplet $(2, t, t)$ is sum-feasible if and only if $2 \mid t$. In case of the product we have the following result.

Theorem 8. The triplet $(2, t, t) \in \mathbb{N}^{3}$ is product-feasible if and only if $2 \mid t$ or $3 \mid t$.

The paper is organized as follows. In Section 2 we prove auxiliary results and some necessary conditions for a triplet to be sum-feasible or compositum-feasible. Section 3 contains some explicit constructions for Table 1 as well as the proofs of Theorems 6 and 7. In Section 4 we prove Theorem 8 and provide impossibility proofs which are used in the proof of Theorem 5 later on. The proof of Theorem 5 is divided into two parts. At the end of Section 3 we prove that each triplet given in Table 1 is sum-feasible. The proof that no other triplets are sum-feasible is given at the end of Section 4.

\section{Lemmas}

Lemma 9 (Part of [23, Lemma 1]). Let $\alpha_{1}, \alpha_{2}, \alpha_{3}$ be distinct conjugate algebraic numbers. Then $\alpha_{2} \pm \alpha_{3} \neq \pm 2 \alpha_{1}$ for all four choices of signs \pm . 
Let $\alpha_{1}, \alpha_{2}, \ldots, \alpha_{n}$ be the roots of a nonzero separable polynomial $f(x) \in \mathbb{Q}[x]$ of degree $n \geqslant 2$. An additive relation between $\alpha_{1}, \alpha_{2}, \ldots, \alpha_{n}$ is a relation of the kind

$$
a_{1} \alpha_{1}+a_{2} \alpha_{2}+\cdots+a_{n} \alpha_{n} \in \mathbb{Q},
$$

where all the $a_{j} \in \mathbb{Q}$. We call this additive relation trivial if $a_{1}=a_{2}=$ $\cdots=a_{n}$.

Recall that the Galois group $G$ of $f$ is 2-transitive if for any two pairs of the roots of $f$, say, $\alpha, \alpha^{\prime}, \alpha \neq \alpha^{\prime}$, and $\alpha_{i}, \alpha_{j}, \alpha_{i} \neq \alpha_{j}$, there is an automorphism $\sigma \in G$ such that $\sigma(\alpha)=\alpha_{i}$ and $\sigma\left(\alpha^{\prime}\right)=\alpha_{j}$.

Lemma 10 (Part of Theorem 3 in [1] - see also [24]). Suppose that the Galois group of a separable polynomial $f(x) \in \mathbb{Q}[x]$ of degree $n$ is 2 -transitive. Then there are no nontrivial additive relations between the roots of $f$.

For a polynomial of prime degree we have the following.

Lemma 11 (Special case of [5, Theorem 2]). There are no nontrivial additive relations between the roots $\alpha_{1}, \alpha_{2}, \ldots, \alpha_{p}$ of an irreducible polynomial $f(x) \in \mathbb{Q}[x]$ of prime degree $p$.

Lemma 12 ([16, Theorem 1.12]). If $K$ and $L$ are number fields and $K / \mathbb{Q}$ is Galois then

$$
[K L: \mathbb{Q}]=\frac{[K: \mathbb{Q}] \cdot[L: \mathbb{Q}]}{[K \cap L: \mathbb{Q}]} .
$$

Lemma $13([\mathbf{2 1}])$. Suppose that $\alpha$ is a root of an irreducible polynomial $f(x) \in \mathbb{Q}[x]$. Let $r$ be the number of linear factors of $f(x)$ over $\mathbb{Q}(\alpha)$. Then $r$ divides the degree of $f(x)$.

As usual, denote by $\operatorname{lcm}(a, b)$ and $\operatorname{gcd}(a, b)$ the least common multiple and the greatest common divisor of positive integers $a$ and $b$, respectively.

Lemma 14. Suppose that a triplet $(a, b, c)$ is sum-feasible, productfeasible or compositum-feasible. Then $c \mid \operatorname{lcm}(a, b) \cdot t$ for some positive integer $t \leqslant \operatorname{gcd}(a, b)$.

Proof: Assume that a triplet $(a, b, c)$ is sum-feasible, product-feasible or compositum-feasible. Then there exist algebraic numbers $\alpha, \beta, \gamma$ or degrees $a, b, c$, respectively, such that $\alpha+\beta+\gamma=0$ or $\alpha \beta \gamma=1$ or $\mathbb{Q}(\alpha, \beta)=\mathbb{Q}(\gamma)$. In any case, it is clear that the degree $D$ of the compositum $\mathbb{Q}(\alpha, \beta)$ is divisible by $\operatorname{lcm}(a, b)$, since $a \mid D$ and $b \mid D$, so that $D=\operatorname{lcm}(a, b) \cdot t$ say. Clearly,

$$
\begin{aligned}
D & =[\mathbb{Q}(\alpha, \beta): \mathbb{Q}] \\
& =[\mathbb{Q}(\alpha, \beta): \mathbb{Q}(\beta)] \cdot[\mathbb{Q}(\beta): \mathbb{Q}] \leqslant[\mathbb{Q}(\alpha): \mathbb{Q}] \cdot[\mathbb{Q}(\beta): \mathbb{Q}]=a b .
\end{aligned}
$$


Hence $t \leqslant a b / \operatorname{lcm}(a, b)=\operatorname{gcd}(a, b)$. Finally, note that $c \mid D$, because $\mathbb{Q}(\gamma)$ is a subfield of $\mathbb{Q}(\alpha, \beta)$.

\section{Constructions}

Let $K$ be a number field of degree $n$ over $\mathbb{Q}$, with $\mathcal{O}_{K}$ its ring of integers, $d_{K}$ its discriminant, and $\sigma_{1}, \ldots, \sigma_{n}$ be the $n$ distinct $\mathbb{Q}$-invariant embeddings of $K$ into $\mathbb{C}$. If $\alpha$ is an arbitrary element of $K$ then its discriminant, which we shall denote by $d_{K}(\alpha)$, is defined by

$$
d_{K}(\alpha)=\prod_{1 \leqslant i<j \leqslant n}\left(\sigma_{i}(\alpha)-\sigma_{j}(\alpha)\right)^{2} .
$$

It is well-known that if $\alpha \in \mathcal{O}_{K}$ then $d_{K}(\alpha)$ is a rational integer which is divisible by $d_{K}$ (see [20, Proposition 2.13]).

Lemma 15 ([19, Exercise 4.5.4 and solution]). If $\alpha$ is a root of an irreducible polynomial $x^{n}+a x+b \in \mathbb{Z}[x], n \geqslant 2$, then

$$
d_{K}(\alpha)=(-1)^{n(n-1) / 2}\left(n^{n} b^{n-1}+(-1)^{n-1}(n-1)^{n-1} a^{n}\right)
$$

where $K=\mathbb{Q}(\alpha)$.

Lemma 16. For any positive integers $n$ and $D$ there exists an extension $K / \mathbb{Q}$ of degree $n$ whose discriminant $d_{K}$ is coprime to $D$.

Proof: If $D=1$ then one can take any number field $K$ of degree $n$. If $n=1$ then one can take $K=\mathbb{Q}$, since $d_{\mathbb{Q}}=1$. So we can assume that $D \geqslant 2$ and $n \geqslant 2$.

Suppose that the set of primes that divide $D$ is $\left\{p_{1}, p_{2}, \ldots, p_{r}, q_{1}\right.$, $\left.q_{2}, \ldots, q_{s}\right\}$ where $p_{i} \mid n$ and $q_{j} \nmid n$ for $i=1, \ldots, r$ and $j=1, \ldots, s$. Choose a prime number $q$ such that

$$
q>\max \left\{p_{1}, \ldots, p_{r}, q_{1}, \ldots, q_{s}\right\} .
$$

Eisenstein's Criterion implies the irreducibility of the polynomial

$$
x^{n}+q q_{1} \cdots q_{s} x+q .
$$

Let $\alpha$ be any root of this polynomial. Then, by Lemma 15, we obtain

$$
d_{\mathbb{Q}(\alpha)}(\alpha)=(-1)^{n(n-1) / 2} \cdot q^{n-1}\left(n^{n}+(-1)^{n-1}(n-1)^{n-1} q\left(q_{1} \cdots q_{s}\right)^{n}\right) .
$$

It is easy to see that the number $d_{\mathbb{Q}(\alpha)}(\alpha)$ is coprime to $p_{1} \cdots p_{r} q_{1} \cdots q_{s}$, and therefore coprime to $D$. Hence the discriminant of the number field $K=\mathbb{Q}(\alpha)$, which is a divisor of $d_{K}(\alpha)$, is coprime to $D$. 
Lemma 17 ([10, Theorem 87], [19, Exercise 6.5.14 and solution]). If $K$ and $L$ are number fields, of degrees $m$ and $n$, respectively, whose discriminants are coprime numbers, then their compositum is a field of degree $m n$.

Lemma 18 (Part of [10, Theorem 88]). If $K$ and $L$ are number fields of degrees $m$ and $n$, respectively, with coprime discriminants $d_{K}$ and $d_{L}$, respectively, then the discriminant of their compositum $K L$ is $d_{K}^{n} d_{L}^{m}$.

Proposition 19. For any positive integers $a$ and $b$ the triplet $(a, b, a b)$ is compositum-feasible and hence both sum-feasible and product-feasible.

Proof: Let $K$ be a number field of degree $a$. By Lemma 16, there exists an extension $L / \mathbb{Q}$ of degree $b$ whose discriminant $d_{L}$ is coprime to $d_{K}$. By Lemma 17 , we have $[K L: \mathbb{Q}]=a b$, and hence $(a, b, a b)$ is compositumfeasible. By Proposition 1, the triplet $(a, b, a b)$ is both sum-feasible and product-feasible.

Lemma 20. Suppose that $\alpha$ and $\beta$ are algebraic numbers and that $\beta$ is of the same degree $d$ over $\mathbb{Q}$ and over $\mathbb{Q}(\alpha)$. Then for any conjugate $\alpha^{\prime}$ of $\alpha$ the degree of $\beta$ over $\mathbb{Q}\left(\alpha^{\prime}\right)$ is also $d$.

Proof: Assume that $\beta$ has degree $n, 1 \leqslant n<d$, over the field $\mathbb{Q}\left(\alpha^{\prime}\right)$, where $\alpha^{\prime}$ is a conjugate of $\alpha$. Then $\beta$ is a root of a polynomial $P$ of degree $n$ with coefficients in $\mathbb{Q}\left(\alpha^{\prime}\right)$. Take an automorphism $\sigma$ of the Galois group of $\mathbb{Q}(\alpha, \beta) / \mathbb{Q}$ which maps $\alpha^{\prime}$ to $\alpha$. It maps $P$ to a polynomial of degree $n$ with coefficients in $\mathbb{Q}(\alpha)$ whose root is $\sigma(\beta)$. So the conjugate $\beta^{\prime}=\sigma(\beta)$ of $\beta$ over $\mathbb{Q}$ has degree at most $n$ over $\mathbb{Q}(\alpha)$, a contradiction.

Proposition 21. Suppose that $\alpha$ and $\beta$ are algebraic numbers of degrees $m$ and $n$ over $\mathbb{Q}$, respectively. Let $\alpha_{1}=\alpha, \alpha_{2}, \ldots, \alpha_{m}$ be the distinct conjugates of $\alpha$, and let $\beta_{1}=\beta, \beta_{2}, \ldots, \beta_{n}$ be the distinct conjugates of $\beta$. If $\beta$ is of degree $n$ over $\mathbb{Q}(\alpha)$ then all the numbers $\alpha_{i}+\beta_{j}$ (resp. $\alpha_{i} \beta_{j}$ ), $1 \leqslant i \leqslant m, 1 \leqslant j \leqslant n$, are conjugate over $\mathbb{Q}$ (although not necessarily distinct).

Proof: Since $\beta$ is of degree $n$ over $\mathbb{Q}(\alpha)$, for any $j, 1 \leqslant j \leqslant n$, there exists an automorphism of the Galois group of $\mathbb{Q}(\alpha, \beta) / \mathbb{Q}$ which fixes $\alpha$ and maps $\beta$ to $\beta_{j}$. Hence all the numbers $\alpha+\beta_{j}\left(\right.$ resp. $\left.\alpha \beta_{j}\right), 1 \leqslant j \leqslant n$, are conjugate over $\mathbb{Q}$.

Note that $[\mathbb{Q}(\alpha, \beta): \mathbb{Q}]=[\mathbb{Q}(\alpha): \mathbb{Q}] \cdot[\mathbb{Q}(\alpha, \beta): \mathbb{Q}(\alpha)]=m n$, and therefore $\alpha$ is of degree $m$ over $\mathbb{Q}(\beta)$. By Lemma $20, \alpha$ is of degree $m$ over $\mathbb{Q}\left(\beta_{j}\right)$ for any $j, 1 \leqslant j \leqslant n$. Now fix $j, 1 \leqslant j \leqslant n$. For any $i, 1 \leqslant i \leqslant$ 
$m$, there exists an automorphism of the Galois group of $\mathbb{Q}(\alpha, \beta) / \mathbb{Q}$ which fixes $\beta_{j}$ and maps $\alpha$ to $\alpha_{i}$. Hence all $m n$ numbers $\alpha_{i}+\beta_{j}\left(\operatorname{resp} . \alpha_{i} \beta_{j}\right)$, where $1 \leqslant i \leqslant m$ and $1 \leqslant j \leqslant n$, are conjugate over $\mathbb{Q}$.

Lemma 22. Suppose that $p$ is a prime number and $u, v, w$ are nonnegative integers such that $\max (u, v) \leqslant w \leqslant u+v$. Then for any positive integer $D$ there exist number fields $K$ and $L$ of degrees $p^{u}$ and $p^{v}$, respectively, such that the degree of the compositum $K L$ is $p^{w}$ and the discriminant $d_{K L}$ of $K L$ is coprime to $D$.

Proof: Set $C=u+v-w, A=w-v$ and $B=w-u$, so that $A \geqslant 0$, $B \geqslant 0, C \geqslant 0$. By Lemma 16 , there exist number fields $K_{1}, L_{1}$ and $M$ of degrees $p^{C}, p^{A}$ and $p^{B}$, respectively, such that

$$
\begin{aligned}
& \operatorname{gcd}\left(d_{K_{1}}, D\right)=1, \\
& \operatorname{gcd}\left(d_{L_{1}}, D \cdot d_{K_{1}}\right)=1, \\
& \operatorname{gcd}\left(d_{M}, D \cdot d_{K_{1}} \cdot d_{L_{1}}\right)=1 .
\end{aligned}
$$

Then, by Lemma 17, we have

$$
\begin{aligned}
{\left[K_{1} L_{1}: \mathbb{Q}\right] } & =p^{C} \cdot p^{A}=p^{u}, \\
{\left[K_{1} M: \mathbb{Q}\right] } & =p^{C} \cdot p^{B}=p^{v}, \\
{\left[K_{1} L_{1} M: \mathbb{Q}\right] } & =p^{C} \cdot p^{A} \cdot p^{B}=p^{w} .
\end{aligned}
$$

Put $K=K_{1} L_{1}$ and $L=K_{1} M$. Lemma 18 implies that the discriminant $d_{K L}$ of the number field $K L=K_{1} L_{1} M$ is coprime to $D$.

Note that in Proposition 1 the algebraic numbers $\alpha, \beta$ and the rational number $t$ can be chosen so that $\alpha$ is not a conjugate of $-\alpha, t+\beta$ is not a conjugate of $-t-\beta$ and $\alpha^{-1}(t+\beta)^{-1}$ is not a conjugate of $-\alpha^{-1}(t+\beta)^{-1}$. Combining this argument with Lemma 22, by choosing an appropriate $\alpha$, $\beta$ and $t \in \mathbb{Q}$ in the multiplicative case, we obtain the following.

Corollary 23. Suppose that $p$ is a prime number and $u, v, w$ are nonnegative integers such that $\max (u, v) \leqslant w \leqslant u+v$. Then for any positive integer $D$ there exist algebraic numbers $\alpha, \beta, \gamma$ of degrees $p^{u}, p^{v}, p^{w}$ such that $\alpha+\beta+\gamma=0$ (resp. $\alpha \beta \gamma=1$ ) and the discriminant $d_{\mathbb{Q}(\alpha, \beta)}$ of the number field $\mathbb{Q}(\alpha, \beta)$ is coprime to $D$.

Furthermore, in the multiplicative case, $\alpha \beta \gamma=1$, the numbers $\alpha, \beta$ and $\gamma$ can be chosen so that $-\alpha$ is not a conjugate of $\alpha,-\beta$ is not a conjugate of $\beta$ and $-\gamma$ is not a conjugate of $\gamma$. 
Note that one can also give an explicit construction illustrating Lemma 22 and Corollary 23 assuming that $p$ does not divide $D$. Take $u$ distinct prime numbers $p_{1}, \ldots, p_{u}$ and $v$ distinct prime numbers $q_{1}, \ldots, q_{v}$ so that the first $C=u+v-w$ (where $w$ in the range $\max (u, v) \leqslant w \leqslant u+v$ ) numbers in those sets are the same

$$
p_{1}=q_{1}, \ldots, p_{C}=q_{C},
$$

i.e.,

$$
\left\{p_{1}, p_{2}, \ldots, p_{u}\right\} \cap\left\{q_{1}, q_{2}, \ldots, q_{v}\right\}=\left\{p_{1}, p_{2}, \ldots, p_{C}\right\} .
$$

Assume that the prime numbers $p_{i}(1 \leqslant i \leqslant u)$ and $q_{i}(1 \leqslant i \leqslant v)$ are all greater than $p$ and $D$ and that $p$ does not divide $D$. Set

$$
K=\mathbb{Q}\left(p_{1}^{1 / p}, \ldots, p_{u}^{1 / p}\right), \quad L=\mathbb{Q}\left(q_{1}^{1 / p}, \ldots, q_{v}^{1 / p}\right) .
$$

Then

$$
K L=\mathbb{Q}\left(p_{1}^{1 / p}, \ldots, p_{u}^{1 / p}, q_{C+1}^{1 / p}, \ldots, q_{v}^{1 / p}\right) .
$$

Clearly, $K$ is of degree $p^{u}, L$ is of degree $p^{v}$ and $K L$ is of degree $p^{u} p^{v-C}=$ $p^{w}$ with discriminant coprime to $D$.

To illustrate Corollary 23 we can take

$$
\alpha=p_{1}^{1 / p}+\cdots+p_{u}^{1 / p}, \quad \beta=q_{1}^{1 / p}+\cdots+q_{v}^{1 / p},
$$

and

$$
\gamma=-2 p_{1}^{1 / p}-\cdots-2 p_{C}^{1 / p}-p_{C+1}^{1 / p}-\cdots-p_{u}^{1 / p}-q_{C+1}^{1 / p}-\cdots-q_{v}^{1 / p}
$$

for the sum and

$\alpha=\left(p_{1}^{1 / p}+1\right) \cdots\left(p_{u}^{1 / p}+1\right), \quad \beta=\left(q_{1}^{1 / p}+1\right) \cdots\left(q_{v}^{1 / p}+1\right), \quad \gamma=(\alpha \beta)^{-1}$

for the product. In both cases, $\operatorname{deg}(\alpha)=p^{u}, \operatorname{deg}(\beta)=p^{v}, \operatorname{deg}(\gamma)=$ $p^{u} p^{v-C}=p^{w}$.

Lemma 24. Suppose that $\alpha, \beta, \gamma, \delta, \mu$ and $\nu$ are algebraic numbers of degrees $a, b, c, a^{\prime}, b^{\prime}$ and $c^{\prime}$, respectively, such that $\alpha+\beta+\gamma=0$ (resp. $\alpha \beta \gamma=1$ ) and $\delta+\mu+\nu=0$ (resp. $\delta \mu \nu=1$ and, in addition, $\delta$ is not a conjugate of $-\delta, \mu$ is not a conjugate of $-\mu, \nu$ is not a conjugate of $-\nu)$. If

$$
\operatorname{gcd}\left(d_{\mathbb{Q}(\alpha)}, d_{\mathbb{Q}(\delta)}\right)=\operatorname{gcd}\left(d_{\mathbb{Q}(\beta)}, d_{\mathbb{Q}(\mu)}\right)=\operatorname{gcd}\left(d_{\mathbb{Q}(\gamma)}, d_{\mathbb{Q}(\nu)}\right)=1
$$

then the triplet $\left(a a^{\prime}, b b^{\prime}, c c^{\prime}\right)$ is sum-feasible (resp. product-feasible).

Proof: Let us first deal with the additive case when

$$
\alpha+\beta+\gamma=\delta+\mu+\nu=0 .
$$


Since $\operatorname{gcd}\left(d_{\mathbb{Q}(\alpha)}, d_{\mathbb{Q}(\delta)}\right)=1$, by Lemma 17 , we obtain $[\mathbb{Q}(\alpha, \delta): \mathbb{Q}]=a a^{\prime}$. We claim that

$$
\mathbb{Q}(\alpha, \delta)=\mathbb{Q}(\alpha+\delta) .
$$

Indeed, let $\alpha_{1}, \alpha_{2}, \ldots, \alpha_{a}$ and $\delta_{1}, \delta_{2}, \ldots, \delta_{a^{\prime}}$ be all the distinct conjugates of $\alpha$ and $\delta$, respectively. Without loss of generality we may assume that $a, a^{\prime} \geqslant 2$, since otherwise (2) automatically holds.

By Proposition 21, all the numbers $\alpha_{i}+\delta_{j}, 1 \leqslant i \leqslant a, 1 \leqslant j \leqslant a^{\prime}$, are conjugate. Suppose that $\mathbb{Q}(\alpha, \delta) \neq \mathbb{Q}(\alpha+\delta)$. Then $\alpha_{i}+\delta_{j}=\alpha_{k}+\delta_{l}$ with certain $i \neq k$ and $j \neq l$. So $\alpha_{i}-\alpha_{k}=\delta_{l}-\delta_{j}$. The difference of two distinct conjugates of an algebraic number of degree at least two is irrational, e.g., by trace consideration or by Hilbert's theorem 90 . (See [9] for the description of all algebraic numbers expressible as the difference of two conjugate numbers.) Therefore,

$$
L:=\mathbb{Q}(\alpha)^{\mathrm{Gal}} \cap \mathbb{Q}(\delta)^{\mathrm{Gal}} \neq \mathbb{Q},
$$

where $K^{\mathrm{Gal}}$ denotes the Galois closure of the number field $K$.

Since $L=\mathbb{Q}(\alpha)^{\text {Gal }} \cap \mathbb{Q}(\delta)^{\text {Gal }} \neq \mathbb{Q}$, by Minkowski's theorem, we must have $\left|d_{L}\right|>1$. However, the discriminants of the fields $\mathbb{Q}(\alpha)^{\text {Gal }}$ and $\mathbb{Q}(\delta)^{\text {Gal }}$ are both divisible by $d_{L}$, which is impossible in view of $\operatorname{gcd}\left(d_{\mathbb{Q}(\alpha)}, d_{\mathbb{Q}(\delta)}\right)=1$. (For any prime number $p$ we have $p \mid d_{K}$ if and only if $p \mid d_{K^{\mathrm{Gal}}}$; see [20, p. 159].) This proves (2).

Analogously, we obtain $[\mathbb{Q}(\beta, \mu): \mathbb{Q}]=b b^{\prime}$ and $\mathbb{Q}(\beta, \mu)=\mathbb{Q}(\beta+\mu)$. Also, $[\mathbb{Q}(\gamma, \nu): \mathbb{Q}]=c c^{\prime}$ and $\mathbb{Q}(\gamma, \nu)=\mathbb{Q}(\gamma+\nu)$. Hence

$$
\begin{aligned}
& \operatorname{deg}(\alpha+\delta)=[\mathbb{Q}(\alpha, \delta): \mathbb{Q}]=a a^{\prime}, \\
& \operatorname{deg}(\beta+\mu)=[\mathbb{Q}(\beta, \mu): \mathbb{Q}]=b b^{\prime}, \\
& \operatorname{deg}(\gamma+\nu)=[\mathbb{Q}(\gamma, \nu): \mathbb{Q}]=c c^{\prime}
\end{aligned}
$$

and $(\alpha+\delta)+(\beta+\mu)+(\gamma+\nu)=0$. This completes the proof of additive version of the lemma.

To prove the multiplicative version, where $\alpha \beta \gamma=\delta \mu \nu=1$, we first claim that

$$
\mathbb{Q}(\alpha, \delta)=\mathbb{Q}(\alpha \delta) .
$$

As above, let $\alpha_{1}, \alpha_{2}, \ldots, \alpha_{a}$ and $\delta_{1}, \delta_{2}, \ldots, \delta_{a^{\prime}}$ be all the distinct conjugates of $\alpha$ and $\delta$, respectively. We may also assume that $a, a^{\prime} \geqslant 2$, since otherwise (7) certainly holds.

Suppose that $\mathbb{Q}(\alpha, \delta) \neq \mathbb{Q}(\alpha \delta)$. Then $\alpha_{i} \delta_{j}=\alpha_{k} \delta_{l}$ with some $i \neq k$ and $j \neq l$. So $\alpha_{i} / \alpha_{k}=\delta_{l} / \delta_{j}$. We shall prove that in this case (3) also holds. Indeed, observe that the quotient of two distinct conjugate algebraic numbers is rational if and only if it is a root of unity. The 
only such number distinct from 1 is -1 . So $\delta_{l} / \delta_{j} \in \mathbb{Q}$ if and only if $\delta_{l}=-\delta_{j}$ which is impossible, by our extra assumption that $\delta$ is not a conjugate of $-\delta$. Now, exactly the same argument as above leads to a contradiction and completes the proof of (7).

Next, by the same argument, we must have $[\mathbb{Q}(\beta, \mu): \mathbb{Q}]=b b^{\prime}$ and $\mathbb{Q}(\beta, \mu)=\mathbb{Q}(\beta \mu)$ and also $[\mathbb{Q}(\gamma, \nu): \mathbb{Q}]=c c^{\prime}$ and $\mathbb{Q}(\gamma, \nu)=\mathbb{Q}(\gamma \nu)$. Hence, instead of (4)-(6), we obtain

$$
\begin{aligned}
& \operatorname{deg}(\alpha \delta)=[\mathbb{Q}(\alpha, \delta): \mathbb{Q}]=a a^{\prime}, \\
& \operatorname{deg}(\beta \mu)=[\mathbb{Q}(\beta, \mu): \mathbb{Q}]=b b^{\prime}, \\
& \operatorname{deg}(\gamma \nu)=[\mathbb{Q}(\gamma, \nu): \mathbb{Q}]=c c^{\prime}
\end{aligned}
$$

and $(\alpha \delta) \cdot(\beta \mu) \cdot(\gamma \nu)=1$.

Remark 25. In fact, if $\alpha, \beta, \gamma, \delta, \mu$ and $\nu$ are algebraic numbers of degrees $a, b, c, a^{\prime}, b^{\prime}$ and $c^{\prime}$, respectively, such that $\alpha+\beta+\gamma=0$ and $\delta+\mu+\nu=0$ and if

$$
[\mathbb{Q}(\alpha, \delta)]=a a^{\prime}, \quad[\mathbb{Q}(\beta, \mu)]=b b^{\prime} \quad \text { and } \quad[\mathbb{Q}(\gamma, \nu)]=c c^{\prime}
$$

then there is a rational number $t$ such that

$$
\operatorname{deg}(\alpha+t \delta)=a a^{\prime}, \quad \operatorname{deg}(\beta+t \mu)=b b^{\prime} \quad \text { and } \quad \operatorname{deg}(\gamma+t \nu)=c c^{\prime} .
$$

Since $(\alpha+t \delta)+(\beta+t \mu)+(\gamma+t \nu)=0$, the triplet $\left(a a^{\prime}, b b^{\prime}, c c^{\prime}\right)$ is sum-feasible.

Lemma 26. Suppose that $K_{1}, L_{1}, K_{2}, L_{2}$ are number fields of degrees $a_{1}, b_{1}, a_{2}, b_{2}$, respectively. Let $c_{1}=\left[K_{1} L_{1}: \mathbb{Q}\right]$ and $c_{2}=\left[K_{2} L_{2}: \mathbb{Q}\right]$, and suppose that the discriminant $d_{K_{1} L_{1}}$ of the compositum $K_{1} L_{1}$ is coprime to the discriminant $d_{K_{2} L_{2}}$ of $K_{2} L_{2}$. Then the triplet $\left(a_{1} a_{2}, b_{1} b_{2}, c_{1} c_{2}\right)$ is compositum-feasible.

Proof: It is well-known that if $K$ is a subfield of a number field $L$ then $d_{K}$ divides $d_{L}$ (see, e.g., [20, Proposition 2.16]). So the discriminant of any subfield of $K_{1} L_{1}$ is coprime to the discriminant of any subfield of $K_{2} L_{2}$. Hence, by Lemma 17, we have

$$
\begin{aligned}
{\left[K_{1} K_{2}: \mathbb{Q}\right] } & =a_{1} a_{2}, \\
{\left[L_{1} L_{2}: \mathbb{Q}\right] } & =b_{1} b_{2}, \\
{\left[K_{1} L_{1} K_{2} L_{2}: \mathbb{Q}\right] } & =c_{1} c_{2} .
\end{aligned}
$$

Here $K_{1} L_{1} K_{2} L_{2}$ is the compositum of $K_{1} L_{1}$ and $K_{2} L_{2}$ which coincides with the compositum of $K_{1} K_{2}$ and $L_{1} L_{2}$. Therefore the triplet $\left(a_{1} a_{2}, b_{1} b_{2}, c_{1} c_{2}\right)$ is compositum-feasible. 
Corollary 27. Suppose that $p$ is a prime number and $u, v, w$ are nonnegative integers such that $\max (u, v) \leqslant w \leqslant u+v$, and that the triplet $(a, b, c) \in \mathbb{N}^{3}$ is compositum-feasible. Then the triplet $\left(a p^{u}, b p^{v} c p^{w}\right)$ is also compositum-feasible.

Proof: Since the triplet $(a, b, c)$ is compositum-feasible, there exist number fields $K_{1}$ and $L_{1}$ of degrees $a$ and $b$, respectively, such that the degree of the compositum $K_{1} L_{1}$ is $c$. By Lemma 22 with $D=d_{K_{1} L_{1}}$, there exist number fields $K_{2}$ and $L_{2}$ of degrees $p^{u}$ and $p^{v}$, respectively, such that the degree of the compositum $K_{2} L_{2}$ is $p^{w}$ and the discriminant $d_{K_{2} L_{2}}$ of $K_{2} L_{2}$ is coprime to the discriminant $d_{K_{1} L_{1}}$ of $K_{1} L_{1}$. Then Lemma 26 implies that the triplet $\left(a p^{u}, b p^{v}, c p^{w}\right)$ is compositum-feasible.

Proof of Theorem 6: Let $p_{1}<p_{2}<\cdots<p_{s}$ be the primes dividing the product $a b c$. Only $p_{1}$ can be even. Assume that the exponents of $p_{i}$ in $a$, $b, c$ are $u_{i} \geqslant 0, v_{i} \geqslant 0, w_{i} \geqslant 0$, respectively, so that

$$
a=\prod_{i=1}^{s} p_{i}^{u_{i}}, \quad b=\prod_{i=1}^{s} p_{i}^{v_{i}}, \quad c=\prod_{i=1}^{s} p_{i}^{w_{i}} .
$$

We start with $p_{1}$ and, by Corollary 23 with $D=D_{1}=1$, construct the numbers $\alpha_{1}, \beta_{1}, \gamma_{1}$ of degrees $p_{1}^{u_{1}}, p_{1}^{v_{1}}, p_{1}^{w_{1}}$, respectively, such that $\alpha_{1}+\beta_{1}+\gamma_{1}=0$ (resp. $\alpha_{1} \beta_{1} \gamma_{1}=1$ ). Set $D_{2}=d_{\mathbb{Q}\left(\alpha_{1}, \beta_{1}\right)}$. By Corollary 23 with $D=D_{2}$ and Lemma 24, there exist algebraic numbers $\alpha_{2}$, $\beta_{2}, \gamma_{2}$ of degrees $p_{2}^{u_{2}}, p_{2}^{v_{2}}, p_{2}^{w_{2}}$, respectively, such that $\alpha_{2}+\beta_{2}+\gamma_{2}=0$ (resp. $\alpha_{2} \beta_{2} \gamma_{2}=1$ ). Moreover, since $p_{2}$ is odd, $\alpha_{2}, \beta_{2}, \gamma_{2}$ are of odd degree (so the multiplicative version of Lemma 24 is applicable) and the degrees of $\alpha_{1}+\alpha_{2}, \beta_{1}+\beta_{2}, \gamma_{1}+\gamma_{2}$ (resp. $\left.\alpha_{1} \alpha_{2}, \beta_{1} \beta_{2}, \gamma_{1} \gamma_{2}\right)$ are $p_{1}^{u_{1}} p_{2}^{u_{2}}$, $p_{1}^{v_{1}} p_{2}^{v_{2}}, p_{1}^{w_{1}} p_{2}^{w_{2}}$, respectively. Next, selecting $D_{i}=d_{\mathbb{Q}\left(\alpha_{1}, \beta_{1}, \ldots, \alpha_{i-1}, \beta_{i-1}\right)}$ for $i=3, \ldots, s$ and continuing step-by-step in this fashion (by Corollary 23 with $D=D_{i}$ at $i$ th step and Lemma 24) we will end up with the numbers

$$
\alpha=\alpha_{1}+\cdots+\alpha_{s}, \quad \beta=\beta_{1}+\cdots+\beta_{s}, \quad \gamma=\gamma_{1}+\cdots+\gamma_{s}
$$

(resp. $\alpha=\alpha_{1} \cdots \alpha_{s}, \beta=\beta_{1} \cdots \beta_{s}, \gamma=\gamma_{1} \cdots \gamma_{s}$ ) of degrees $\prod_{i=1}^{s} p_{i}^{u_{i}}=a$, $\prod_{i=1}^{s} p_{i}^{v_{i}}=b, \prod_{i=1}^{s} p_{i}^{w_{i}}=c$, respectively, satisfying $\alpha+\beta+\gamma=0$ (resp. $\alpha \beta \gamma=1$.)

Proof of Theorem 7: Assume that the inequality

$$
\max \left\{\operatorname{ord}_{p}(a), \operatorname{ord}_{p}(b)\right\} \leqslant \operatorname{ord}_{p}(c)
$$

holds for every prime number $p$. Now, as above, repeated application of Corollary 27 (for primes dividing $c$ ) implies that the triplet $(a, b, c)$ is compositum-feasible. 
As a consequence of Corollary 23 and Lemma 24, we also state the following proposition (which is a partial case of Conjecture 4).

Proposition 28. Suppose that the triplet $(a, b, c) \in \mathbb{N}^{3}$ satisfies the exponent triangle inequality with respect to any prime number. Then for any sum-feasible (resp. product-feasible) triplet $\left(a^{\prime}, b^{\prime}, c^{\prime}\right) \in \mathbb{N}^{3}$ the triplet $\left(a a^{\prime}, b b^{\prime}, c c^{\prime}\right)$ is also sum-feasible (resp. product-feasible).

Proof: Suppose that the triplet $\left(a^{\prime}, b^{\prime}, c^{\prime}\right)$ is product-feasible. We can start with the triplet $\alpha^{\prime}, \beta^{\prime}, \gamma^{\prime}$ of degrees $a^{\prime}, b^{\prime}, c^{\prime}$ such that $\alpha^{\prime} \beta^{\prime} \gamma^{\prime}=1$ and with $D=d_{\mathbb{Q}\left(\alpha^{\prime}, \beta^{\prime}\right)}$. Then, as above, we apply Corollary 23 and Lemma 24 for each prime dividing $a b c$. The proof in the additive case is the same except that in this case we do not need to use the second part of Corollary 23.

Proposition 29. Suppose that $n \geqslant 2$ is a positive integer.

(i) The triplets $(n, n, n)$ and $(n, n, n(n-1))$ are compositum-feasible.

(ii) The triplet $(n, n, n(n-1) / 2)$ is sum-feasible and product-feasible, but if $n$ is even then it is not compositum-feasible.

(iii) The triplet $(n, n, 2 n)$ is compositum-feasible.

Proof: (i) Take $K$ to be an arbitrary number field of degree $n$ over $\mathbb{Q}$. Then the compositum $K K=K$ also has degree $n$. Therefore the triplet $(n, n, n)$ is compositum-feasible for every $n \in \mathbb{N}$.

Let $\alpha$ and $\alpha^{\prime}$ be two distinct conjugate algebraic numbers of degree $n$ such that the Galois group of their minimal polynomial is the symmetric group $S_{n}$. We claim that the degree of $\alpha^{\prime}$ over $\mathbb{Q}(\alpha)$ equals $n-1$. Indeed, we have $\left[\mathbb{Q}\left(\alpha, \alpha^{\prime}\right): \mathbb{Q}\right]=[\mathbb{Q}(\alpha): \mathbb{Q}] \cdot\left[\mathbb{Q}\left(\alpha, \alpha^{\prime}\right): \mathbb{Q}(\alpha)\right] \leqslant n(n-1)$. On the other hand, if the degree of $\alpha^{\prime}$ over the field $\mathbb{Q}(\alpha)$ were less than $n-1$ then the degree of the splitting field of the minimal polynomial of $\alpha$ is less than $n$ !, a contradiction. So $\left[\mathbb{Q}\left(\alpha, \alpha^{\prime}\right): \mathbb{Q}\right]=n(n-1)$, and therefore $(n, n, n(n-1))$ is compositum-feasible. Of course, combining this with Proposition 1 , we also have that the triplets $(n, n, n), n \in \mathbb{N}$, and $(n, n, n(n-1)), n \geqslant 2$, are both sum-feasible and product-feasible.

(ii) Let $\alpha$ be an algebraic number of degree $n$ such that the Galois group of its minimal polynomial is $S_{n}$. Let $\alpha_{1}=\alpha, \alpha_{2}, \ldots, \alpha_{n}$ be distinct conjugates of $\alpha$ over $\mathbb{Q}$. Consider the following set

$$
A=\left\{\alpha_{i}+\alpha_{j} \mid i, j=1,2, \ldots, n, i<j\right\} .
$$


Each element of $A$ is a conjugate of $\alpha_{1}+\alpha_{2}$, because $S_{n}$ is 2-transitive. If two numbers of $A$, say $\alpha_{i}+\alpha_{j}$ and $\alpha_{k}+\alpha_{l}$ with either $i \neq k$ or $j \neq l$, were equal, then we would have a nontrivial additive relation between the conjugates of $\alpha$, which is impossible in view of Lemma 10. So the set $A$ contains exactly $n(n-1) / 2$ distinct numbers, and therefore the triplet $(n, n, n(n-1) / 2)$ is sum-feasible.

For the product we cannot use Lemma 10 directly. Nevertheless, by the same argument, considering the set

$$
A_{1}=\left\{\alpha_{i} \alpha_{j} \mid i, j=1,2, \ldots, n, i<j\right\}
$$

we will deduce that the triplet $(n, n, n(n-1) / 2)$ is product-feasible. Indeed, assume that $\alpha_{i} \alpha_{j}=\alpha_{k} \alpha_{l}$, where $\{i, j\} \neq\{k, l\}$. We have an immediate contradiction, unless the list $\alpha_{i}, \alpha_{j}, \alpha_{k}, \alpha_{l}$ contains four distinct numbers. In this latter case, we must have $n \geqslant 4$. Since the Galois group of $\mathbb{Q}(\alpha)$ over $\mathbb{Q}$ contains the transposition $(i, l)$, from $\alpha_{i} \alpha_{j}=$ $\alpha_{k} \alpha_{l}$ we obtain $\alpha_{l} \alpha_{j}=\alpha_{k} \alpha_{i}$. Thus $\alpha_{i}^{2}=\alpha_{l}^{2}$. Since $\alpha_{i} \neq \alpha_{l}$, this implies $\alpha_{i}=-\alpha_{l}$, which is impossible by Lemma 10 .

Note that if a triplet $(a, b, c)$ is compositum-feasible then $a \mid c$ and $b \mid c$. However, $n$ does not divide $n(n-1) / 2$ for even $n \geqslant 2$. Hence for $n$ even the triplet $(n, n, n(n-1) / 2)$ is not compositum-feasible.

(iii) If $n$ is even then the triplet $(n, n, 2 n)$ is compositum-feasible, by Theorem 7 .

Now suppose that $n>1$ is odd. Let $p>2$ be a prime number dividing $n$. Proposition 5.5.2 of [13] implies that the dihedral group $D_{p}$ can be realized as a Galois group of a number field over $\mathbb{Q}$, i.e., there exists a number field $L$ of degree $2 p$ over $\mathbb{Q}$ whose Galois group is isomorphic to the dihedral group $D_{p}$ (also see [14], [18]). Let $H$ be a subgroup of $D_{p}$ of order 2 and let $K \subset L$ be the fixed field of $H$. By the primitive element theorem, $K=\mathbb{Q}(\theta)$ for some algebraic number $\theta$ of degree $p$. The extension $K / \mathbb{Q}$ is not Galois, because $H$ is not a normal subgroup of $D_{p}$. So there is a conjugate $\theta^{\prime}$ of $\theta$ such that $L=\mathbb{Q}\left(\theta, \theta^{\prime}\right)$. Therefore, the triplet $(p, p, 2 p)$ is compositum-feasible.

Let $p_{1}, p_{2}, \ldots, p_{k}$ be the set of primes (not necessarily distinct) dividing $n / p$, so that $n / p=p_{1} p_{2} \cdots p_{k}$. Since the triplet $(p, p, 2 p)$ is compositum-feasible, by Corollary 27 , the triplet $\left(p p_{1}, p p_{1}, 2 p p_{1}\right)$ is also compositum-feasible. Now, the repeated application of Corollary 27 (for prime numbers $\left.p_{2}, p_{3}, \ldots, p_{k}\right)$ implies that the triplet $\left(p p_{1} \cdots p_{k}, p p_{1} \cdots p_{k}\right.$, $\left.2 p p_{1} \cdots p_{k}\right)=(n, n, 2 n)$ is compositum-feasible. 
Proposition 29 can be generalized as follows. Let $\theta_{1}, \theta_{2}, \ldots, \theta_{n}$ be distinct conjugate algebraic numbers of degree $n \geqslant 2$ (over $\mathbb{Q}$ ) such that the Galois group of their minimal polynomial is the full symmetric group $S_{n}$. We shall say that the triplet $(a, b, c) \in \mathbb{N}^{3}$ is symmetrically generated if there exist algebraic numbers $\alpha, \beta$ and $\gamma$ of degrees $a, b$ and $c$, respectively, such that $\alpha+\beta+\gamma=0$ and both $\alpha$ and $\beta$ (and hence $\gamma$ too) are linear forms in conjugates of $\theta$ (of degree $n$ with Galois group $S_{n}$ ), i.e., there exist $x_{i}, y_{i} \in \mathbb{Z}, i=1,2, \ldots, n$, such that

$$
\begin{aligned}
\alpha & =x_{1} \theta_{1}+x_{2} \theta_{2}+\cdots+x_{n} \theta_{n}, \\
\beta & =y_{1} \theta_{1}+y_{2} \theta_{2}+\cdots+y_{n} \theta_{n} .
\end{aligned}
$$

Suppose that $\alpha$ is a linear form in conjugates of $\theta$, i.e., there exists a function $f:\{1,2, \ldots, n\} \rightarrow \mathbb{Z}$ such that

$$
\alpha=f(1) \cdot \theta_{1}+f(2) \cdot \theta_{2}+\cdots+f(n) \cdot \theta_{n} .
$$

Let

$$
A_{f}=\{f(1), f(2), \ldots, f(n)\} \backslash\{0\}, \quad m_{f}=\left|A_{f}\right|,
$$

and for $j \in A_{f}$ let $k_{j}$ be the number of indices $i$ in $\{1,2, \ldots, n\}$ for which $f(i)=j$. If $A_{f}=\emptyset$ then $\alpha=0$, and therefore $\operatorname{deg}(\alpha)=1$. Suppose that $A_{f} \neq \emptyset$. Then in view of Lemma 10 it is easy to see that the degree of $\alpha$ $($ over $\mathbb{Q}$ ) is

$$
\operatorname{deg}(\alpha)=\frac{n \cdot(n-1) \cdots\left(n-m_{f}+1\right)}{\prod_{j \in A_{f}} k_{j} !} .
$$

Similarly, writing

$$
\beta=g(1) \cdot \theta_{1}+g(2) \cdot \theta_{2}+\cdots+g(n) \cdot \theta_{n}
$$

and

$$
\gamma=h(1) \cdot \theta_{1}+h(2) \cdot \theta_{2}+\cdots+h(n) \cdot \theta_{n},
$$

where $h(x)=-f(x)-g(x)$, we find that

$$
\operatorname{deg}(\beta)=\frac{n \cdot(n-1) \cdots\left(n-m_{g}+1\right)}{\prod_{j \in A_{g}} k_{j} !}
$$

and

$$
\operatorname{deg}(\gamma)=\frac{n \cdot(n-1) \cdots\left(n-m_{h}+1\right)}{\prod_{j \in A_{h}} k_{j} !}
$$

The triplet $(\operatorname{deg}(\alpha), \operatorname{deg}(\beta), \operatorname{deg}(\gamma))$ given in $(8)-(10)$ is symmetrically generated for any functions $f, g:\{1,2, \ldots, n\} \rightarrow \mathbb{Z}$. 
Consider an example with $\alpha=\theta_{1}$ and $\beta=\theta_{2}$. Then $\alpha$ and $\beta$ are both of degree $n$ while the degree of $\gamma=-(\alpha+\beta)=-\theta_{1}-\theta_{2}$ is $n(n-1) / 2$. Analogously, $\alpha=\theta_{1}$ and $\beta=2 \theta_{2}$ are both of degree $n$ while the degree of $\gamma=-(\alpha+\beta)=-\theta_{1}-2 \theta_{2}$ is $n(n-1)$. So both triplets $(n, n, n(n-1) / 2)$ and $(n, n, n(n-1))$ are symmetrically generated (see Proposition 29 ).

In order to get some new symmetrically generated triplets let us fix $i, j \in \mathbb{N}$ satisfying $i+j \leqslant n$ and take

$\alpha=x_{1} \theta_{1}+\cdots+x_{i} \theta_{i}, \quad \beta=x_{i+1} \theta_{i+1}+\cdots+x_{i+j} \theta_{i+j}, \quad \gamma=-(\alpha+\beta)$.

Selecting $x_{1}=x_{2}=\cdots=x_{i+j}=1$ we find that the triplet

$$
\left(\left(\begin{array}{c}
n \\
i
\end{array}\right),\left(\begin{array}{c}
n \\
j
\end{array}\right),\left(\begin{array}{c}
n \\
i+j
\end{array}\right)\right)
$$

is symmetrically generated. Selecting $x_{k}=k$ for $k=1, \ldots, i+j$ we deduce that the triplet

$$
\left(i !\left(\begin{array}{c}
n \\
i
\end{array}\right), j !\left(\begin{array}{c}
n \\
j
\end{array}\right),(i+j) !\left(\begin{array}{c}
n \\
i+j
\end{array}\right)\right)
$$

is symmetrically generated.

Lemma $30([\mathbf{3}])$. Suppose that $\alpha$ is a root of an irreducible trinomial $f(x)=x^{n}+a x+b \in \mathbb{Z}[x]$. Let $\Delta(f)$ be the discriminant of $f$, i.e.,

$$
\Delta(f)=(-1)^{n(n-1) / 2}\left(n^{n} b^{n-1}+(-1)^{n-1}(n-1)^{n-1} a^{n}\right) .
$$

If $\operatorname{gcd}(n, a)=\operatorname{gcd}(a(n-1), b)=1$ and $|\Delta(f)|$ is not the square of an integer then the Galois group of $f$ is the full symmetric group $S_{n}$.

Proposition 31. For any positive integers $n>1$ and $D$ there exists a number field $K$ of degree $n$ (over $\mathbb{Q}$ ) whose normal closure $L$ has Galois group isomorphic to the full symmetric group $S_{n}$ and whose discriminant $d_{L}$ of $L / \mathbb{Q}$ is coprime to $D$.

Proof: Suppose that the set of primes that divide $D$ is

$$
\left\{p_{1}, p_{2}, \ldots, p_{r}, q_{1}, q_{2}, \ldots, q_{s}\right\},
$$

where $p_{i} \mid n$ and $q_{j} \nmid n$ for $i=1, \ldots, r$ and $j=1, \ldots, s$. Set $a=q_{1} q_{2} \cdots q_{s}$ if $s \geqslant 1$ and $a=1$ otherwise. Consider the polynomial

$$
f(x)=x^{n}+a x+q \in \mathbb{Z}[x],
$$

where $q$ is a sufficiently large prime number. 
Note that this condition guarantees the irreducibility of $f(x)$ over $\mathbb{Q}$. Indeed, assume that $f(x)$ is reducible over $\mathbb{Q}$, so $f(x)=u(x) v(x)$ with $u(x), v(x) \in \mathbb{Z}[x], \operatorname{deg}(u), \operatorname{deg}(v) \geqslant 1$. Since $q$ is a prime number we have either $u(0)= \pm 1$ or $v(0)= \pm 1$. So $f(x)$ has a root $x_{0}$ such that $\left|x_{0}\right| \leqslant 1$. But then

$$
q=\left|-a x_{0}-x_{0}^{n}\right| \leqslant|a| \cdot\left|x_{0}\right|+\left|x_{0}\right|^{n} \leqslant a+1 \leqslant q_{1} q_{2} \cdots q_{s}+1,
$$

which is false for $q$ sufficiently large.

By Lemma 15 and the choice of $a$ and $q$, the discriminant

$$
\Delta(f)=(-1)^{n(n-1) / 2}\left(n^{n} q^{n-1}+(-1)^{n-1}(n-1)^{n-1} a^{n}\right)
$$

of $f(x)$ is coprime to $D$. Suppose that $\alpha$ is a root of $f(x)$ and $K=$ $\mathbb{Q}(\alpha)$. It is well known that the discriminant $d_{K}$ of $K$ divides $\Delta(f)$ (see Proposition 2.13 in $[\mathbf{2 0}]$ ). Therefore, $d_{K}$ is also coprime to $D$.

It remains to show that there exists a prime $q$ for which the Galois group of the normal closure of $K$ is isomorphic to the full symmetric group $S_{n}$.

Clearly, if $n=2$ then the extension $K / \mathbb{Q}$ is normal and its Galois group is $S_{2}$. Suppose that $n=3$. Then

$$
|\Delta(f)|=27 q^{2}+4 a^{3} \equiv 3 \quad(\bmod 4),
$$

because $q$ is odd. Hence $|\Delta(f)|$ is not the square of an integer. Now, Lemma 30 implies that the Galois group of the polynomial $f(x)$ (and hence the Galois group of the normal closure of $K$ ) is $S_{3}$.

Assume that $n \geqslant 4$. We claim that for each sufficiently large prime number $q$ the Galois group of the normal closure of $K$ is isomorphic to the full symmetric group $S_{n}$. Let us check the the conditions of Lemma 30. Clearly the condition

$$
\operatorname{gcd}(n, a)=\operatorname{gcd}(a(n-1), q)=1
$$

of Lemma 30 is satisfied for $q$ sufficiently large. So it remains only to prove that for each sufficiently large $q$ the number

$$
|\Delta(f)|=n^{n} q^{n-1}+(-1)^{n-1}(n-1)^{n-1} a^{n}
$$

is not the square of an integer.

Consider the curve

$$
y^{2}=A x^{n-1}+B,
$$

where $A=n^{n}$ and $B=(-1)^{n-1}(n-1)^{n-1} a^{n}$. The polynomial $A x^{n-1}+$ $B \in \mathbb{Z}[x]$ is separable, and therefore the genus of the curve (11) is at least 1. By a well-known theorem of Siegel (see [22]), the curve (11) has a finite number of integer points. If the curve (11) does have integer 
solutions, let $\left(x_{1}, y_{1}\right)$ be the one with largest $\left|x_{1}\right|$. Set $x_{1}=1$ if the curve (11) has no integer solutions. Then for any positive integer $q>x_{1}$ the number $|\Delta(f)|$ is not the square of an integer. Now, selecting a sufficiently large prime number $q$ we see that the conditions of Lemma 30 are satisfied. So there exists a number field $K=\mathbb{Q}(\alpha)$ of degree $n$ whose normal closure $L$ has Galois group isomorphic to $S_{n}$ and the discriminant $d_{K}$ of $K / \mathbb{Q}$ is coprime to $D$.

Finally, as we already observed above, by [20, p. 159], if $p$ is a prime number then $p \mid d_{K}$ if and only if $p \mid d_{L}$. Hence $d_{L}$ is coprime to $D$.

In addition to Proposition 28 we obtain one more special case of Conjecture 4 (see Section 1).

Proposition 32. Suppose that the triplet $(a, b, c) \in \mathbb{N}^{3}$ is sum-feasible. Then for any symmetrically generated triplet $\left(a^{\prime}, b^{\prime}, c^{\prime}\right) \in \mathbb{N}^{3}$ the triplet $\left(a a^{\prime}, b b^{\prime}, c c^{\prime}\right)$ is also sum-feasible.

Proof: Fix any algebraic numbers $\alpha, \beta, \gamma$ of degrees $a, b, c$ such that $\alpha+\beta+\gamma=0$. Suppose that $D$ is the discriminant of the field $\mathbb{Q}(\alpha, \beta, \gamma)=$ $\mathbb{Q}(\alpha, \beta)$. Since the triplet $\left(a^{\prime}, b^{\prime}, c^{\prime}\right)$ is symmetrically generated, there is an $n \in \mathbb{N}$ and algebraic numbers $\alpha^{\prime}, \beta^{\prime}, \gamma^{\prime}$ of degrees $a^{\prime}, b^{\prime}, c^{\prime}$ such that $\alpha^{\prime}+\beta^{\prime}+\gamma^{\prime}=0$ and $\alpha^{\prime}$ and $\beta^{\prime}$ are linear forms in conjugates of an algebraic number $\theta$ of degree $n \geqslant 2$ whose Galois group is $S_{n}$.

By Proposition 31, there is a number field $K$ of degree $n$ (over $\mathbb{Q}$ ) whose normal closure $L$ has the Galois group isomorphic to the full symmetric group $S_{n}$ and the discriminant $d_{L}$ of $L$ is coprime to $D$. We can take $K=\mathbb{Q}(\theta)$ and then select $\alpha^{\prime}, \beta^{\prime}, \gamma^{\prime}$ of degrees $a^{\prime}, b^{\prime}, c^{\prime}$ as linear forms in conjugates of $\theta$. Applying Lemmas 17 and 24, we find that the degrees of $\alpha+\alpha^{\prime}, \beta+\beta^{\prime}$ and $\gamma+\gamma^{\prime}$ are $a a^{\prime}, b b^{\prime}$ and $c c^{\prime}$, respectively, whereas their sum is zero.

Proof of Theorem 5 (constructions): We first prove that the triplets displayed in Table 1 are compositum-feasible except for

$$
(4,4,6), \quad(4,6,6), \quad(6,6,8), \quad(6,6,9), \quad(6,6,15) .
$$

There are 40 such triplets. Then we show that the triplets $(4,4,6)$, $(4,6,6),(6,6,9)$ and $(6,6,15)$ are sum-feasible. (The triplet $(6,6,8)$ is left undecided here, but it is shown to be not sum feasible in [6].) The proof that no other triplets are sum-feasible is given at the end of the next section. 
Theorem 7 shows that the following 33 triplets, given in Table 2, are compositum-feasible.

TABle 2. Compositum-feasible triplets from Theorem 7.

\begin{tabular}{ccccccc}
\hline $\mathbf{b} \backslash \mathbf{a}$ & $\mathbf{1}$ & $\mathbf{2}$ & $\mathbf{3}$ & $\mathbf{4}$ & $\mathbf{5}$ & $\mathbf{6}$ \\
\hline $\mathbf{1}$ & 1 & & & & & \\
\hline $\mathbf{2}$ & 2 & 2,4 & & & & \\
\hline $\mathbf{3}$ & 3 & 6 & 3,9 & & & \\
\hline $\mathbf{4}$ & 4 & 4,8 & 12 & $4,8,16$ & & \\
\hline $\mathbf{5}$ & 5 & 10 & 15 & 20 & 5,25 & \\
\hline $\mathbf{6}$ & 6 & 6,12 & 6,18 & 12,24 & 30 & 6,12, \\
& & & & & & 18,36 \\
\hline
\end{tabular}

The triplets $(3,3,6),(4,4,12),(5,5,20)$ and $(6,6,30)$ are compositumfeasible, by Proposition 29. Since the triplet $(3,3,6)$ is compositumfeasible, Corollary 27 implies that the triplets $(3,6,12)$ and $(6,6,24)$ are both compositum-feasible. The triplet $(5,5,10)$ is compositum-feasible, by Proposition 29 (iii). This gives $33+4+2+1=40$ compositum-feasible triplets.

So all the triplets of Table 1 are compositum-feasible except for

$$
(4,4,6), \quad(4,6,6), \quad(6,6,8), \quad(6,6,9), \quad(6,6,15) .
$$

(If $a \nmid c$ or $b \nmid c$ then the triplet $(a, b, c)$ is not compositum-feasible.) This completes the proof of the compositum part of Theorem 5 .

For the sum-feasible part of Theorem 5, note that, by Proposition 1, if the triplet is compositum-feasible then it is sum-feasible as well. So all the triplets of Table 1, except possibly

$$
(4,4,6), \quad(4,6,6), \quad(6,6,8), \quad(6,6,9), \quad(6,6,15),
$$

are sum-feasible. It remains to show that the triplets

$$
(4,4,6), \quad(4,6,6), \quad(6,6,9), \quad(6,6,15)
$$

are sum-feasible, the triplet $(6,6,8)$ being left undecided. Indeed, the triplets $(4,4,6)$ and $(6,6,15)$ are sum-feasible, by Proposition 29 (ii), while the triplets $(4,6,6)$ and $(6,6,9)$ are sum-feasible, by Theorem 6 .

The proof that the remaining triplets (those not in Table 1) are not sum-feasible is given at the end of the next section. 


\section{Impossibility proofs}

We first prove Theorem 8 . Then we show that the four special cases from the set

$$
\mathcal{S}=\{(3,6,9),(4,6,8),(5,5,15),(6,6,10)\}
$$

are not sum-feasible. At the end of the section we will complete the proof of Theorem 5 by showing that each triplet $(a, b, c), a \leqslant b \leqslant c, b \leqslant 6$ that is not in Table 1 is not sum-feasible.

Proof of Theorem 8: Let $t=2 n$, where $n \in \mathbb{N}$, and put $\alpha=\sqrt{-1}$ and $\beta=\sqrt[t]{2}$. Then $\alpha \beta$ is conjugate to $\beta$, and so of degree $t$. Hence the triplet $(2,2 n, 2 n)$ is product-feasible. Similarly, for $t=3 n, \alpha=e^{2 \pi i / 3}$ and $\beta=\sqrt[t]{2}, \alpha \beta$ is again conjugate to $\beta$, and so of degree $t$. Hence the triplet $(2,3 n, 3 n)$ is product-feasible.

Suppose next that $t$ is a positive integer that is not divisible by 2 or by 3 . Assume that the triplet $(2, t, t)$ is product-feasible. Clearly $t>1$. Then there exist three algebraic numbers $\alpha, \beta$ and $\gamma$ of degrees $2, t$ and $t$, respectively, such that $\alpha \beta=\gamma$. The degree $[\mathbb{Q}(\alpha, \beta): \mathbb{Q}]$ is divisible by 2 and by $t$, because $\mathbb{Q}(\alpha)$ and $\mathbb{Q}(\beta)$ are the subfields of $\mathbb{Q}(\alpha, \beta)$. On the other hand,

$[\mathbb{Q}(\alpha, \beta): \mathbb{Q}]=[\mathbb{Q}(\alpha, \beta): \mathbb{Q}(\alpha)] \cdot[\mathbb{Q}(\alpha): \mathbb{Q}] \leqslant[\mathbb{Q}(\alpha): \mathbb{Q}] \cdot[\mathbb{Q}(\beta): \mathbb{Q}]=2 t$.

So $[\mathbb{Q}(\alpha, \beta): \mathbb{Q}]=2 t$ and we have the following diagram.

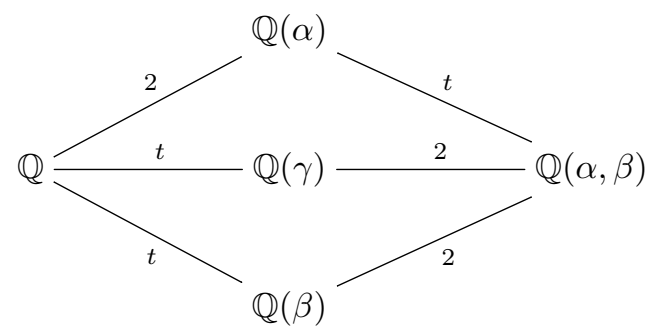

Let $\beta_{1}=\beta, \beta_{2}, \ldots, \beta_{t}$ be the distinct conjugates of $\beta$ over $\mathbb{Q}$. From the diagram we see that $\beta$ is of degree $t$ over $\mathbb{Q}(\alpha)$. Hence for every $j \in\{1,2, \ldots, t\}$ there exists an automorphism $\sigma_{j}$ in the Galois group of the normal closure of $\mathbb{Q}(\alpha, \beta, \gamma)=\mathbb{Q}(\alpha, \beta)$ over $\mathbb{Q}$ which fixes $\alpha$ and 
sends $\beta=\beta_{1}$ to $\beta_{j}$. On applying $\sigma_{j}, j=1, \ldots, t$, to $\alpha \beta=\gamma$ we obtain

$$
\begin{gathered}
\alpha \beta_{1}=\gamma_{1}, \\
\alpha \beta_{2}=\gamma_{2}, \\
\vdots \\
\alpha \beta_{t}=\gamma_{t},
\end{gathered}
$$

where $\gamma_{j}=\sigma_{j}(\gamma), 1 \leqslant j \leqslant t$. Then all the conjugates $\gamma_{j}, 1 \leqslant j \leqslant t$, are distinct. (If $\gamma_{i}=\gamma_{j}$ with $i \neq j$ then (13) implies $\beta_{i}=\beta_{j}$, which is not the case.) On multiplying together all the equalities in (13) we obtain $\alpha^{t}=r \in \mathbb{Q}$, because the numbers $\beta_{1} \beta_{2} \cdots \beta_{t}$ and $\gamma_{1} \gamma_{2} \cdots \gamma_{t}$ are absolute norms of $\beta$ and $\gamma$, respectively. It follows that the quadratic algebraic number $\alpha$ is a root of a polynomial $p(x)=x^{t}-r$ for some $r \in \mathbb{Q}$. Let $\theta=r^{1 / t}$ be the real root of $p(x)$ (recall that $t$ is odd and $>1$ ) and let $\varepsilon=e^{2 \pi i / t}$ be the primitive th root of unity. Then all the roots of $p(x)$ are

$$
\theta, \theta \varepsilon, \theta \varepsilon^{2}, \ldots, \theta \varepsilon^{t-1} .
$$

Let $\alpha^{\prime} \neq \alpha$ be the (only) conjugate of $\alpha$ over $\mathbb{Q}$. Then $\alpha^{\prime}$ also is a root of $p(x)$. So $\alpha=\theta \varepsilon^{k}$ and $\alpha^{\prime}=\theta \varepsilon^{l}$ with certain $k, l \in\{0,1, \ldots, t-1\}$, $k \neq l$. We claim that $l=t-k$. Indeed, note that $\alpha \alpha^{\prime}=\theta^{2} \varepsilon^{k+l} \in \mathbb{Q}$. Since $\theta^{2}$ is real, so is $\varepsilon^{k+l}$. Hence $\varepsilon^{k+l}= \pm 1$. This yields $k+l \in\{t / 2, t, 3 t / 2\}$, since $0<k+l<2 t$. Therefore, $k+l=t$, because $t$ is odd. This implies

$$
\alpha=\theta \varepsilon^{k}, \quad \alpha^{\prime}=\theta \varepsilon^{t-k}=\theta \varepsilon^{-k} .
$$

It follows that $\alpha+\alpha^{\prime}=\theta\left(\varepsilon^{k}+\varepsilon^{-k}\right) \in \mathbb{Q}$ and $\alpha \alpha^{\prime}=\theta^{2} \in \mathbb{Q}$. Combining this with $\theta^{t} \in \mathbb{Q}$, where $t$ is odd, we deduce that $\theta \in \mathbb{Q}$. Hence $\varepsilon^{k}+\varepsilon^{-k}=$ $\left(\alpha+\alpha^{\prime}\right) / \theta \in \mathbb{Q}$. The number $\varepsilon^{k}+\varepsilon^{-k}=2 \cos (2 \pi k / t)$ is an algebraic integer, so it must be a rational integer. Consequently,

$$
\varepsilon^{k}+\varepsilon^{-k}=2 \cos (2 \pi k / t) \in\{0, \pm 1, \pm 2\} .
$$

(See also $[\mathbf{1 2}]$ and $[\mathbf{2 5}]$.)

If $2 \cos (2 \pi k / t)=0$ then $2 \pi k / t=\pi / 2+\pi s$ for some $s \in \mathbb{Z}$. This yields $4 k=t(2 s+1)$ which is impossible, because $t$ is odd. Next, $2 \cos (2 \pi k / t)=$ \pm 1 implies that $t$ is divisible by 3 , which is not the case. Finally, if $2 \cos (2 \pi k / t)= \pm 2$ then $2 \pi k / t=\pi s$ for some $s \in \mathbb{Z}$. So $\varepsilon^{k}=\varepsilon^{-k}=$ $(-1)^{s}$, and therefore $\alpha=\theta \varepsilon^{k}=\theta \varepsilon^{-k}=\alpha^{\prime}$, a contradiction.

Now, step by step, we give all necessary impossibility proofs for Theorem 5 .

Theorem 33. The triplet $(3,6,9)$ is not sum-feasible. 
Proof: Suppose that $(3,6,9)$ is sum-feasible. Then there exist algebraic numbers $\alpha, \beta, \gamma$ of degrees 3, 6, 9, respectively, such that $\alpha+\beta+\gamma=0$. The degree of $\mathbb{Q}(\alpha, \beta)$ over $\mathbb{Q}$ is divisible by 9 , because $\mathbb{Q}(\gamma)=\mathbb{Q}(\alpha+\beta)$ is a subfield of $\mathbb{Q}(\alpha, \beta)$. Similarly, $[\mathbb{Q}(\alpha, \beta)$ : $\mathbb{Q}]$ is divisible by 6 , because $\mathbb{Q}(\beta) \subset \mathbb{Q}(\alpha, \beta)$. On the other hand, $[\mathbb{Q}(\alpha, \beta): \mathbb{Q}] \leqslant[\mathbb{Q}(\alpha): \mathbb{Q}] \cdot[\mathbb{Q}(\beta)$ : $\mathbb{Q}]=18$. Hence $[\mathbb{Q}(\alpha, \beta): \mathbb{Q}]=18$ and we have the following diagram.

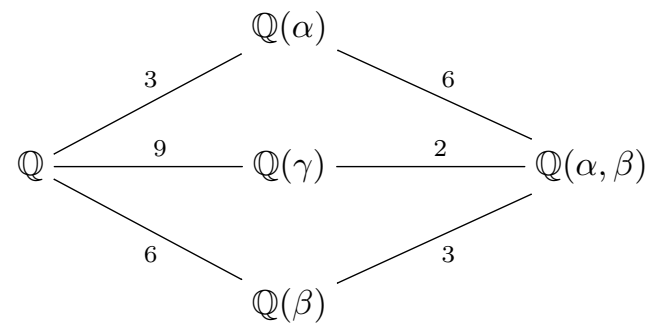

Let $\alpha_{1}, \alpha_{2}, \alpha_{3}$ be the distinct conjugates of $\alpha$ over $\mathbb{Q}$, and let $\beta_{1}, \beta_{2}$, $\beta_{3}, \beta_{4}, \beta_{5}, \beta_{6}$ be the distinct conjugates of $\beta$ over $\mathbb{Q}$. By the diagram, $\beta$ is of degree 6 over $\mathbb{Q}(\alpha)$. Proposition 21 implies that all 18 (not necessarily distinct) numbers $\alpha_{i}+\beta_{j}, 1 \leqslant i \leqslant 3,1 \leqslant j \leqslant 6$, are conjugate over $\mathbb{Q}$. Put

$$
\Gamma_{i}=\left\{\alpha_{i}+\beta_{1}, \alpha_{i}+\beta_{2}, \alpha_{i}+\beta_{3}, \alpha_{i}+\beta_{4}, \alpha_{i}+\beta_{5}, \alpha_{i}+\beta_{6}\right\}, \quad i=1,2,3 .
$$

Clearly, $\left|\Gamma_{i}\right|=6$ for $i=1,2,3$, because all six elements of the set $\Gamma_{i}$ are distinct.

We next claim that $\left|\Gamma_{1} \cap \Gamma_{2}\right|=3$. Indeed, if $\left|\Gamma_{1} \cap \Gamma_{2}\right|<3$ then

$$
\left|\Gamma_{1} \cup \Gamma_{2}\right|=\left|\Gamma_{1}\right|+\left|\Gamma_{2}\right|-\left|\Gamma_{1} \cap \Gamma_{2}\right|=12-\left|\Gamma_{1} \cap \Gamma_{2}\right|>9
$$

which is impossible, because $\gamma=-(\alpha+\beta)$ is of degree 9 over $\mathbb{Q}$.

On the other hand, if $\left|\Gamma_{1} \cap \Gamma_{2}\right| \geqslant 4$ then there exist distinct indices $j_{1}$, $j_{2}, j_{3}, j_{4}$ and distinct indices $k_{1}, k_{2}, k_{3}, k_{4}$ such that

$$
\begin{aligned}
& \alpha_{1}+\beta_{j_{1}}=\alpha_{2}+\beta_{k_{1}}, \\
& \alpha_{1}+\beta_{j_{2}}=\alpha_{2}+\beta_{k_{2}}, \\
& \alpha_{1}+\beta_{j_{3}}=\alpha_{2}+\beta_{k_{3}}, \\
& \alpha_{1}+\beta_{j_{4}}=\alpha_{2}+\beta_{k_{4}},
\end{aligned}
$$

and $\left\{j_{1}, j_{2}, j_{3}, j_{4}, k_{1}, k_{2}, k_{3}, k_{4}\right\} \subseteq\{1,2,3,4,5,6\}$. Evidently,

$$
\left\{j_{1}, j_{2}, j_{3}, j_{4}\right\} \cap\left\{k_{1}, k_{2}, k_{3}, k_{4}\right\} \neq \emptyset \text {. }
$$


Assume without loss of generality that $j_{1}=k_{2}\left(j_{1}=k_{1}\right.$ would imply $\alpha_{1}=\alpha_{2}$, which is not the case). Subtracting (15) from (14) we get

$$
2 \beta_{j_{1}}=\beta_{k_{1}}+\beta_{j_{2}} .
$$

If $k_{1}=j_{2}$ then $j_{1}=k_{1}$ and (14) implies $\alpha_{1}=\alpha_{2}$, which is impossible. So $\beta_{j_{1}}, \beta_{k_{1}}, \beta_{j_{2}}$ are distinct conjugates. But then (16) contradicts Lemma 9. This proves the inequality

$$
\left|\Gamma_{1} \cap \Gamma_{2}\right| \leqslant 3
$$

and so completes the proof of $\left|\Gamma_{1} \cap \Gamma_{2}\right|=3$. Analogously, $\left|\Gamma_{1} \cap \Gamma_{3}\right|=3$ and $\left|\Gamma_{2} \cap \Gamma_{3}\right|=3$.

Since $\left|\Gamma_{1} \cap \Gamma_{2}\right|=3$, after re-indexing the numbers $\beta_{1}, \beta_{2}, \beta_{3}, \beta_{4}, \beta_{5}$, $\beta_{6}$, if necessary, we can write

$$
\begin{aligned}
& \alpha_{1}+\beta_{1}=\alpha_{2}+\beta_{j_{1}}, \\
& \alpha_{1}+\beta_{2}=\alpha_{2}+\beta_{j_{2}}, \\
& \alpha_{1}+\beta_{3}=\alpha_{2}+\beta_{j_{3}} .
\end{aligned}
$$

The indices $j_{1}, j_{2}, j_{3}$ in these equations are distinct. We claim that the set $\left\{j_{1}, j_{2}, j_{3}\right\}$ coincides with $\{4,5,6\}$. Indeed, assume the contrary, i.e., $\left\{j_{1}, j_{2}, j_{3}\right\} \cap\{1,2,3\} \neq \emptyset$. Then without loss of generality we can assume that $j_{1}=2$. Then (18) implies $\alpha_{1}-\alpha_{2}=\beta_{2}-\beta_{1}$, whereas (19) implies $\alpha_{1}-\alpha_{2}=\beta_{j_{2}}-\beta_{2}$. So $\beta_{2}-\beta_{1}=\beta_{j_{2}}-\beta_{2}$, and therefore $2 \beta_{2}=\beta_{1}+\beta_{j_{2}}$, contradicting Lemma 9 .

Now, since $\left\{j_{1}, j_{2}, j_{3}\right\}=\{4,5,6\}$, after re-indexing the numbers $\beta_{4}$, $\beta_{5}, \beta_{6}$, if necessary, we obtain

$$
\begin{aligned}
& \alpha_{1}+\beta_{1}=\alpha_{2}+\beta_{4}, \\
& \alpha_{1}+\beta_{2}=\alpha_{2}+\beta_{5}, \\
& \alpha_{1}+\beta_{3}=\alpha_{2}+\beta_{6} .
\end{aligned}
$$

Similarly, since $\left|\Gamma_{1} \cap \Gamma_{3}\right|=3$, we must have

$$
\begin{aligned}
& \alpha_{1}+\beta_{i_{1}}=\alpha_{3}+\beta_{j_{1}}, \\
& \alpha_{1}+\beta_{i_{2}}=\alpha_{3}+\beta_{j_{2}}, \\
& \alpha_{1}+\beta_{i_{3}}=\alpha_{3}+\beta_{j_{3}},
\end{aligned}
$$

with $\left\{i_{1}, i_{2}, i_{3}, j_{1}, j_{2}, j_{3}\right\}=\{1,2,3,4,5,6\}$. We claim that $\left\{i_{1}, i_{2}, i_{3}\right\}=$ $\{4,5,6\}$. Indeed, if, say $i_{1} \in\{1,2,3\}$ then $\alpha_{1}+\beta_{i_{1}} \in \Gamma_{1} \cap \Gamma_{2} \cap \Gamma_{3}$. 
However, this is impossible, because

$$
\begin{aligned}
\left|\Gamma_{1} \cap \Gamma_{2} \cap \Gamma_{3}\right|= & \left|\Gamma_{1} \cup \Gamma_{2} \cup \Gamma_{3}\right|-\left|\Gamma_{1}\right|-\left|\Gamma_{2}\right|-\left|\Gamma_{3}\right|+\left|\Gamma_{1} \cap \Gamma_{2}\right| \\
& +\left|\Gamma_{1} \cap \Gamma_{3}\right|+\left|\Gamma_{2} \cap \Gamma_{3}\right| \\
= & 9-6-6-6+3+3+3=0 .
\end{aligned}
$$

So $i_{1} \in\{4,5,6\}$. Analogously, $i_{2}, i_{3} \in\{4,5,6\}$, so that $\left\{i_{1}, i_{2}, i_{3}\right\}=$ $\{4,5,6\}$. Consequently, $\left\{j_{1}, j_{2}, j_{3}\right\}=\{1,2,3\}$ and, after rearranging the equalities in (22), we obtain

$$
\begin{aligned}
& \alpha_{1}+\beta_{4}=\alpha_{3}+\beta_{k_{1}}, \\
& \alpha_{1}+\beta_{5}=\alpha_{3}+\beta_{k_{2}}, \\
& \alpha_{1}+\beta_{6}=\alpha_{3}+\beta_{k_{3}},
\end{aligned}
$$

where $\left\{k_{1}, k_{2}, k_{3}\right\}=\{1,2,3\}$.

Finally, by adding all six equalities in (21) and (23), we obtain

$$
6 \alpha_{1}+\sum_{i=1}^{6} \beta_{i}=3 \alpha_{2}+3 \alpha_{3}+\sum_{i=1}^{6} \beta_{i} .
$$

Thus $2 \alpha_{1}=\alpha_{2}+\alpha_{3}$, which contradicts Lemma 9 .

Remark 34. Recall that the triplet $(3,2,3)$ is product-feasible (see Section 1 and Theorem 8$)$. The triplet $(1,3,3)$ satisfies the exponent triangle inequality with respect to any prime number. Hence $(3,6,9)=$ $(3,2,3) \cdot(1,3,3)$ is product-feasible, by Proposition 28 . Since 6 does not divide 9 , the triplet $(3,6,9)$ is not compositum-feasible.

Theorem 35. The triplet $(4,6,8)$ is not sum-feasible.

Proof: Suppose that $(4,6,8)$ is sum-feasible, so that there exist algebraic numbers $\alpha, \beta, \gamma$ of degrees $4,6,8$, respectively, such that $\alpha+\beta+\gamma=0$. The degree of $\mathbb{Q}(\alpha, \beta)$ over $\mathbb{Q}$ is divisible by 8 , because $\mathbb{Q}(\gamma)=\mathbb{Q}(\alpha+\beta)$ is a subfield of $\mathbb{Q}(\alpha, \beta)$. Similarly, $[\mathbb{Q}(\alpha, \beta): \mathbb{Q}]$ is divisible by 6 , because $\mathbb{Q}(\beta) \subset \mathbb{Q}(\alpha, \beta)$. On the other hand, $[\mathbb{Q}(\alpha, \beta): \mathbb{Q}] \leqslant[\mathbb{Q}(\alpha): \mathbb{Q}] \cdot[\mathbb{Q}(\beta)$ : $\mathbb{Q}]=24$. Hence $[\mathbb{Q}(\alpha, \beta): \mathbb{Q}]=24$ and we have the following diagram.

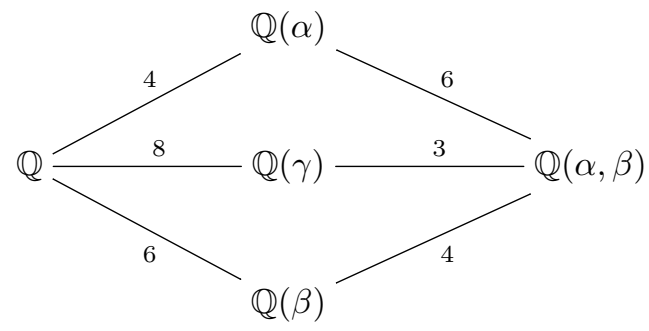


Let $\alpha_{1}, \alpha_{2}, \alpha_{3}, \alpha_{4}$ be the distinct conjugates of $\alpha$ over $\mathbb{Q}$. Similarly, let $\beta_{1}, \beta_{2}, \beta_{3}, \beta_{4}, \beta_{5}, \beta_{6}$ be the distinct conjugates of $\beta$ over $\mathbb{Q}$. By the diagram, $\beta$ is of degree 6 over $\mathbb{Q}(\alpha)$. By Proposition 21, all 24 (not necessarily distinct) numbers $\alpha_{i}+\beta_{j}, 1 \leqslant i \leqslant 4,1 \leqslant j \leqslant 6$, are conjugate over $\mathbb{Q}$. Set

$\Gamma_{i}=\left\{\alpha_{i}+\beta_{1}, \alpha_{i}+\beta_{2}, \alpha_{i}+\beta_{3}, \alpha_{i}+\beta_{4}, \alpha_{i}+\beta_{5}, \alpha_{i}+\beta_{6}\right\}, \quad i=1,2,3,4$. We have $\left|\Gamma_{1} \cup \Gamma_{2} \cup \Gamma_{3} \cup \Gamma_{4}\right|=8$, because the number $\alpha+\beta=-\gamma$ is of degree 8 over $\mathbb{Q}$.

If $\left|\Gamma_{1} \cap \Gamma_{2}\right| \leqslant 3$ then

$8=\left|\Gamma_{1} \cup \Gamma_{2} \cup \Gamma_{3} \cup \Gamma_{4}\right| \geqslant\left|\Gamma_{1} \cup \Gamma_{2}\right|=\left|\Gamma_{1}\right|+\left|\Gamma_{2}\right|-\left|\Gamma_{1} \cap \Gamma_{2}\right| \geqslant 6+6-3=9$, a contradiction. Hence $\left|\Gamma_{1} \cap \Gamma_{2}\right| \geqslant 4$. However, then we get a contradiction in exactly the same way as in the proof of Theorem 33. (See the proof of inequality (17); the degree of $\beta$ over $\mathbb{Q}$ is 6 as in Theorem 33, and we only use two distinct conjugates of $\alpha$, i.e., $\alpha_{1}$ and $\alpha_{2}$.)

Theorem 36. The triplet $(5,5,15)$ is not compositum-feasible.

Proof: It is known (see $[\mathbf{4}$, p. 60]) that the Galois group of the splitting field of an irreducible polynomial of degree 5 is one of the following:

TABLE 3

\begin{tabular}{ccc} 
Group & Generators & Order \\
\hline$S_{5}$ & $(12345),(12)$ & 120 \\
$A_{5}$ & $(12345),(123)$ & 60 \\
$\operatorname{AGL}_{1}(5)$ & $(12345),(2354)$ & 20 \\
$\mathrm{ASL}_{1}(5)$ & $(12345),(25)(34)$ & 10 \\
$C_{5}$ & $(12345)$ & 5 \\
\hline
\end{tabular}

Assume the contrary, i.e., that the triplet $(5,5,15)$ is compositumfeasible. Then there exist algebraic numbers $\alpha$ and $\beta$ such that $[\mathbb{Q}(\alpha)$ : $\mathbb{Q}]=[\mathbb{Q}(\beta): \mathbb{Q}]=5$ and $[\mathbb{Q}(\alpha, \beta): \mathbb{Q}]=15$.

Denote by $K$ and $L$ the Galois closures of $\mathbb{Q}(\alpha)$ and $\mathbb{Q}(\beta)$ over $\mathbb{Q}$, respectively. If neither the Galois group of $K$ nor the Galois group of $L$ is in $\left\{A_{5}, S_{5}\right\}$ then both numbers $[K: \mathbb{Q}]$ and $[L: \mathbb{Q}]$ are in $\{5,10,20\}$. Then, by Lemma 12, the degree of the compositum

$$
[K L: \mathbb{Q}]=\frac{[K: \mathbb{Q}] \cdot[L: \mathbb{Q}]}{[K \cap L: \mathbb{Q}]}
$$


is not divisible by 3 . This is impossible, because the compositum $K L$ has a subfield $\mathbb{Q}(\alpha, \beta)$ of degree 15 over $\mathbb{Q}$. So either $K / \mathbb{Q}$ or $L / \mathbb{Q}$ has Galois group in $\left\{A_{5}, S_{5}\right\}$.

Assume without loss of generality that the Galois group of $K / \mathbb{Q}$ is $A_{5}$ or $S_{5}$. Now $[\mathbb{Q}(\alpha, \beta): \mathbb{Q}]=15$ implies that $\alpha$ is cubic over $\mathbb{Q}(\beta)$ and $\beta$ is cubic over $\mathbb{Q}(\alpha)$. Let $P(x) \in \mathbb{Q}[x]$ be the minimal polynomial of $\alpha$ over $\mathbb{Q}$. We have two possibilities:

(a) Two conjugates of $\alpha$ lie in $\mathbb{Q}(\beta)$, i.e.,

$$
P(x)=\left(x-\alpha^{\prime}\right)\left(x-\alpha^{\prime \prime}\right)\left(x^{3}+a x^{2}+b x+c\right)
$$

where $\alpha^{\prime}, \alpha^{\prime \prime} \in \mathbb{Q}(\beta)$ and $x^{3}+a x^{2}+b x+c \in \mathbb{Q}(\beta)[x]$ is irreducible over $\mathbb{Q}(\beta)$.

(b) No conjugate of $\alpha$ belongs to $\mathbb{Q}(\beta)$, i.e.,

$$
P(x)=\left(x^{2}+a x+b\right)\left(x^{3}+c x^{2}+d x+e\right),
$$

where both polynomials $x^{2}+a x+b \in \mathbb{Q}(\beta)[x]$ and $x^{3}+c x^{2}+d x+e \in$ $\mathbb{Q}(\beta)[x]$ are irreducible over $\mathbb{Q}(\beta)$.

Assume that (a) holds. Then $\alpha^{\prime}=f(\beta)$ for a certain polynomial $f(x) \in \mathbb{Q}[x]$. So $\mathbb{Q}(\beta)$ has a subfield $\mathbb{Q}\left(\alpha^{\prime}\right)=\mathbb{Q}(f(\beta))$ which is of degree 5 over $\mathbb{Q}$. Thus $\mathbb{Q}\left(\alpha^{\prime}\right)=\mathbb{Q}(\beta)$. Then $P(x)$ has exactly two linear factors over $\mathbb{Q}\left(\alpha^{\prime}\right)$, contradicting Lemma 13 .

Suppose now that (b) holds. Denote the Galois group of $K / \mathbb{Q}$ by $G$. Recall that $G=A_{5}$ or $S_{5}$. Assume that $G$ acts on the set $\left\{\alpha_{1}, \alpha_{2}, \alpha_{3}, \alpha_{4}, \alpha_{5}\right\}$ of distinct conjugates of $\alpha$ as follows: if $\tau$ is a permutation of $G$ then $\tau\left(\alpha_{j}\right)=\alpha_{\tau(j)}, j=1,2,3,4,5$.

Suppose that $\alpha^{\prime}$ and $\alpha^{\prime \prime}, \alpha^{\prime} \neq \alpha^{\prime \prime}$, are the conjugates of $\alpha$ that are quadratic over $\mathbb{Q}(\beta)$. Then $\alpha^{\prime}+\alpha^{\prime \prime} \in \mathbb{Q}(\beta)$ and there exists a polynomial $f(x) \in \mathbb{Q}[x]$ such that $\alpha^{\prime}+\alpha^{\prime \prime}=f(\beta)$. Since $A_{5}$ and $S_{5}$ are both 2-transitive groups, there exists $\tau \in G$ such that $\tau\left(\alpha^{\prime}\right)=\alpha_{1}$ and $\tau\left(\alpha^{\prime \prime}\right)=\alpha_{2}$. Then

$$
\alpha_{1}+\alpha_{2}=f\left(\beta_{1}\right)
$$

where $\beta_{1}=\tau(\beta)$ is a conjugate of $\beta$ over $\mathbb{Q}$. On applying the automorphisms id, (23)(45), (24)(35), (25)(34), (123), (124) $\in A_{5} \subseteq G$ to (24) 
we obtain

$$
\begin{aligned}
\alpha_{1}+\alpha_{2} & =f\left(\beta_{1}\right), \\
\alpha_{1}+\alpha_{3} & =f\left(\beta_{2}\right), \\
\alpha_{1}+\alpha_{4} & =f\left(\beta_{3}\right), \\
\alpha_{1}+\alpha_{5} & =f\left(\beta_{4}\right), \\
\alpha_{2}+\alpha_{3} & =f\left(\beta_{5}\right), \\
\alpha_{2}+\alpha_{4} & =f\left(\beta_{6}\right),
\end{aligned}
$$

where $\beta_{1}, \beta_{2}, \beta_{3}, \beta_{4}, \beta_{5}, \beta_{6}$ are some conjugates of $\beta$. Since $\beta$ is of degree 5 over $\mathbb{Q}$ then $\beta_{i}=\beta_{j}$ for some $i \neq j$. Then $i$ th and $j$ th lines of (25) imply that there is a nontrivial additive relation connecting at most 4 conjugates of $\alpha$, contradicting Lemma 11.

Corollary 37. The triplet $(5,5,15)$ is neither sum-feasible nor productfeasible.

Proof: Suppose that $(5,5,15)$ is either sum-feasible or product-feasible, with algebraic numbers $\alpha, \beta, \gamma$ of degrees $5,5,15$, respectively, such that $\alpha+\beta+\gamma=0$ or $\alpha \beta \gamma=1$. In both cases, the degree of $\mathbb{Q}(\alpha, \beta)$ over $\mathbb{Q}$ is divisible by 15 , because $\mathbb{Q}(\gamma)$ is a subfield of $\mathbb{Q}(\alpha, \beta)$. On the other hand, $[\mathbb{Q}(\alpha, \beta): \mathbb{Q}] \leqslant[\mathbb{Q}(\alpha): \mathbb{Q}] \cdot[\mathbb{Q}(\beta): \mathbb{Q}]=25$. So $[\mathbb{Q}(\alpha, \beta): \mathbb{Q}]=15$, contradicting Theorem 36 .

Theorem 38. The triplet $(6,6,10)$ is not sum-feasible.

Proof: Suppose that $(6,6,10)$ is sum-feasible, with algebraic numbers $\alpha$, $\beta, \gamma$ of degrees $6,6,10$, respectively, such that $\alpha+\beta+\gamma=0$. The degree of $\mathbb{Q}(\alpha, \beta)$ over $\mathbb{Q}$ is divisible by 10 , because $\mathbb{Q}(\gamma)=\mathbb{Q}(\alpha+\beta)$ is a subfield of $\mathbb{Q}(\alpha, \beta)$. Similarly, $[\mathbb{Q}(\alpha, \beta): \mathbb{Q}]$ is divisible by 6 , because $\mathbb{Q}(\alpha) \subseteq \mathbb{Q}(\alpha, \beta)$. Hence $[\mathbb{Q}(\alpha, \beta): \mathbb{Q}]$ is divisible by 30. On the other hand, $[\mathbb{Q}(\alpha, \beta): \mathbb{Q}] \leqslant[\mathbb{Q}(\alpha): \mathbb{Q}] \cdot[\mathbb{Q}(\beta): \mathbb{Q}]=36$. Consequently, $[\mathbb{Q}(\alpha, \beta): \mathbb{Q}]=30$ and we have the following diagram.

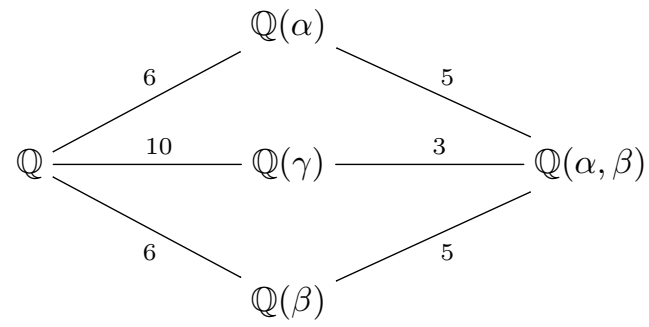


We see that $\beta$ is of degree 5 over $\mathbb{Q}(\alpha)$. Hence $\beta$ has exactly one conjugate, say, $\beta_{1}$, which lies in $\mathbb{Q}(\alpha)$. So $\beta_{1}=f(\alpha)$ for certain polynomial $f(x) \in \mathbb{Q}[x]$ of degree at most 4 . Let $\beta_{1}, \beta_{2}, \beta_{3}, \beta_{4}, \beta_{5}, \beta_{6}$ be all the distinct conjugates of $\beta$ over $\mathbb{Q}$. For every $j=1,2, \ldots, 6$ there exists an automorphism $\sigma_{j}$ of the Galois group of $\mathbb{Q}(\alpha, \beta) / \mathbb{Q}$ which sends $\beta_{1}$ to $\beta_{j}$. On applying $\sigma_{j}$ to $\beta_{1}=f(\alpha)$ we obtain $\beta_{j}=f\left(\alpha_{j}\right), j=1,2, \ldots, 6$. Here $\alpha_{1}=\alpha, \alpha_{2}, \alpha_{3}, \alpha_{4}, \alpha_{5}, \alpha_{6}$ are the (distinct) conjugates of $\alpha$. Assume without loss of generality that $\beta=\beta_{2}=f\left(\alpha_{2}\right)$. Then $-\gamma=\alpha_{1}+f\left(\alpha_{2}\right)$.

The number field $\mathbb{Q}\left(\alpha_{2}\right)$ has a subfield $\mathbb{Q}\left(f\left(\alpha_{2}\right)\right)=\mathbb{Q}\left(\beta_{2}\right)$ of degree 6 over $\mathbb{Q}$. Therefore, $\mathbb{Q}(\beta)=\mathbb{Q}\left(f\left(\alpha_{2}\right)\right)=\mathbb{Q}\left(\alpha_{2}\right)$ and we obtain the following diagram.

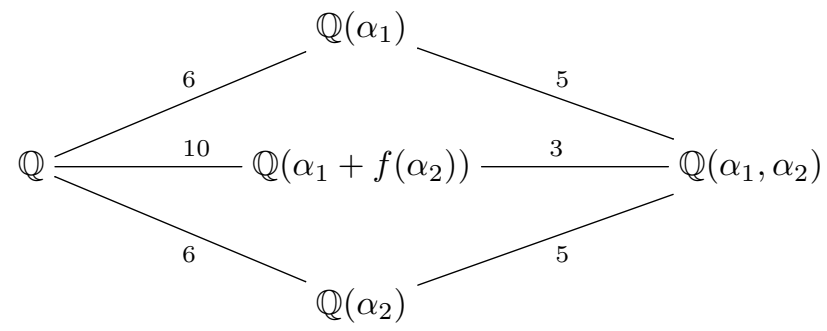

Similarly, $\mathbb{Q}\left(\beta_{j}\right)=\mathbb{Q}\left(f\left(\alpha_{j}\right)\right)=\mathbb{Q}\left(\alpha_{j}\right)$ for $j=1,2, \ldots, 6$. We claim that each $\alpha_{i}$ is of degree 5 over every $\mathbb{Q}\left(\alpha_{j}\right), j \in\{1,2, \ldots, 6\} \backslash\{i\}$. Indeed, fix $j \in\{2,3,4,5,6\}$. Since $\alpha_{2}$ is of degree 5 over $\mathbb{Q}\left(\alpha_{1}\right)$ (see the last diagram), the number $\alpha_{j}$ is conjugate to $\alpha_{2}$ over the field $\mathbb{Q}\left(\alpha_{1}\right)$. Hence there exists an automorphism $\sigma$ of the Galois group of $\mathbb{Q}(\alpha, \beta) / \mathbb{Q}$ which fixes $\alpha_{1}$ and sends $\alpha_{2}$ to $\alpha_{j}$. Thus $\sigma(-\gamma)=\sigma\left(\alpha_{1}+f\left(\alpha_{2}\right)\right)=\alpha_{1}+f\left(\alpha_{j}\right)$ is a conjugate of $-\gamma$. It follows that $\alpha_{1}+f\left(\alpha_{j}\right)$ is of degree 10 over $\mathbb{Q}$, and therefore we have the following diagram. (Recall that, as above, $\left.\mathbb{Q}\left(\beta_{j}\right)=\mathbb{Q}\left(f\left(\alpha_{j}\right)\right)=\mathbb{Q}\left(\alpha_{j}\right).\right)$

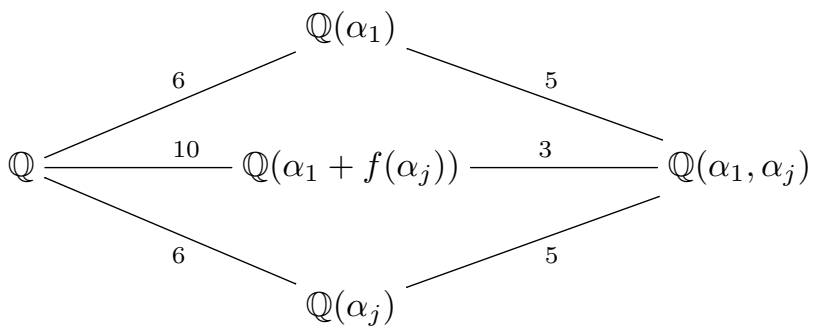

Now, we see that $\alpha_{1}$ is of degree 5 over $\mathbb{Q}\left(\alpha_{j}\right)$ and all the numbers in $\left\{\alpha_{1}, \alpha_{2}, \ldots, \alpha_{6}\right\} \backslash\left\{\alpha_{j}\right\}$ are conjugate to $\alpha_{1}$ over $\mathbb{Q}\left(\alpha_{j}\right)$. Therefore, 
each $\alpha_{i}, i \in\{1,2, \ldots, 6\} \backslash\{j\}$, is of degree 5 over $\mathbb{Q}\left(\alpha_{j}\right)$. Consequently, all the numbers $\alpha_{i}+f\left(\alpha_{j}\right)$, where $i \neq j$, are conjugate over $\mathbb{Q}$.

Consider the following table of numbers which are conjugate to $\alpha_{1}+$ $f\left(\alpha_{2}\right)=-\gamma$.

$$
\begin{aligned}
& \alpha_{1}+f\left(\alpha_{2}\right) \\
& \alpha_{1}+f\left(\alpha_{3}\right) \alpha_{2}+f\left(\alpha_{3}\right) \\
& \alpha_{1}+f\left(\alpha_{4}\right) \alpha_{2}+f\left(\alpha_{4}\right) \alpha_{3}+f\left(\alpha_{4}\right) \\
& \alpha_{1}+f\left(\alpha_{5}\right) \alpha_{2}+f\left(\alpha_{5}\right) \alpha_{3}+f\left(\alpha_{5}\right) \alpha_{4}+f\left(\alpha_{5}\right) \\
& \alpha_{1}+f\left(\alpha_{6}\right) \quad \alpha_{2}+f\left(\alpha_{6}\right) \alpha_{3}+f\left(\alpha_{6}\right) \alpha_{4}+f\left(\alpha_{6}\right) \alpha_{5}+f\left(\alpha_{6}\right) \text {. }
\end{aligned}
$$

The table contains 15 numbers, while the degree of $-\gamma$ over $\mathbb{Q}$ is 10 . Hence

$$
\alpha_{a}+f\left(\alpha_{b}\right)=\alpha_{c}+f\left(\alpha_{t}\right)
$$

with certain $a<b, c<t$ and either $a \neq c$ or $b \neq t$ (because $\operatorname{deg} f \leqslant 4$ ). We claim that $a \neq c$ and $b \neq t$. Indeed, if $b=t$ then $\alpha_{a}=\alpha_{c}$, and therefore $a=c$, which is impossible. Similarly, if $a=c$ then $f\left(\alpha_{b}\right)=$ $f\left(\alpha_{t}\right)$ which implies $\beta_{b}=\beta_{t}$, and hence $b=t$, a contradiction. So $a \neq c$ and $b \neq t$. Assume without loss of generality that $a<c$. Then $a<c<t$, and therefore $t \notin\{a, b, c\}$.

Consider the Galois group $G$ of the normal closure of $\mathbb{Q}\left(\alpha_{1}\right)$ over $\mathbb{Q}$ as acting as a subgroup of $S_{6}$ on the set of indices $\{1,2,3,4,5,6\}$, i.e., if $\sigma \in G$ then $\sigma\left(\alpha_{j}\right)=\alpha_{\sigma(j)}, j=1,2,3,4,5,6$. The order of $G$ is divisible by 5 , because $\left[\mathbb{Q}\left(\alpha_{1}, \alpha_{2}\right): \mathbb{Q}\right]=30$. By Cauchy's Theorem (see, e.g., $[\mathbf{1 7}$, Section 40, Theorem 2]), there exists an automorphism $\tau \in G$ of order 5 in $G$. Then $\tau$ (an element of $S_{6}$ ) is a cycle, say, $\tau=\left(i_{1} i_{2} i_{3} i_{4} i_{5}\right)$ with distinct numbers $i_{1}, i_{2}, i_{3}, i_{4}, i_{5} \in\{1,2,3,4,5,6\}$. Assume without loss of generality that $6 \notin\left\{i_{1}, i_{2}, i_{3}, i_{4}, i_{5}\right\}$. There exists an automorphism $\sigma$ in $G$ which maps $\alpha_{t}$ to $\alpha_{6}$. On applying $\sigma$ to (27) we obtain

$$
\alpha_{i}+f\left(\alpha_{j}\right)=\alpha_{k}+f\left(\alpha_{6}\right)
$$

with $i, j, k \in\{1,2,3,4,5\}$. Now, from (28) and $\tau\left(\alpha_{6}\right)=\alpha_{6}$ we deduce that

$$
\begin{aligned}
& \alpha_{\tau(i)}+f\left(\alpha_{\tau(j)}\right)=\alpha_{\tau(k)}+f\left(\alpha_{6}\right), \\
& \alpha_{\tau^{2}(i)}+f\left(\alpha_{\tau^{2}(j)}\right)=\alpha_{\tau^{2}(k)}+f\left(\alpha_{6}\right), \\
& \alpha_{\tau^{3}(i)}+f\left(\alpha_{\tau^{3}(j)}\right)=\alpha_{\tau^{3}(k)}+f\left(\alpha_{6}\right), \\
& \alpha_{\tau^{4}(i)}+f\left(\alpha_{\tau^{4}(j)}\right)=\alpha_{\tau^{4}(k)}+f\left(\alpha_{6}\right) .
\end{aligned}
$$


The orbits

$$
\begin{aligned}
& \left\{i, \quad \tau(i), \quad \tau^{2}(i), \quad \tau^{3}(i), \quad \tau^{4}(i)\right\}, \\
& \left\{j, \quad \tau(j), \quad \tau^{2}(j), \quad \tau^{3}(j), \quad \tau^{4}(j)\right\}, \\
& \left\{k, \quad \tau(k), \quad \tau^{2}(k), \quad \tau^{3}(k), \quad \tau^{4}(k)\right\}
\end{aligned}
$$

coincide with the set $\{1,2,3,4,5\}$, because $\{i, j, k\} \subset\left\{i_{1}, i_{2}, i_{3}, i_{4}, i_{5}\right\}$. Thus adding (28) and all four equalities of (29) we find that

$$
\sum_{i=1}^{5} \alpha_{i}+\sum_{i=1}^{5} f\left(\alpha_{i}\right)=\sum_{i=1}^{5} \alpha_{i}+5 f\left(\alpha_{6}\right)
$$

and hence

$$
\begin{aligned}
6 \beta_{6}=6 f\left(\alpha_{6}\right) & =f\left(\alpha_{1}\right)+f\left(\alpha_{2}\right)+f\left(\alpha_{3}\right)+f\left(\alpha_{4}\right)+f\left(\alpha_{5}\right)+f\left(\alpha_{6}\right) \\
& =\beta_{1}+\beta_{2}+\beta_{3}+\beta_{4}+\beta_{5}+\beta_{6} \in \mathbb{Q}
\end{aligned}
$$

a contradiction.

Proof of Theorem 5 (impossibility): Recall that if a triplet $(a, b, c) \in \mathbb{N}^{3}$ is sum-feasible then $c \leqslant a b$. Denote by $\mathcal{A}$ the set of triplets $(a, b, c)$ of positive integers satisfying $a \leqslant b \leqslant c, b \leqslant 6$ and $c \leqslant a b$. The set $\mathcal{A}$ contains

$$
\begin{aligned}
\sum_{b=1}^{6} \sum_{a=1}^{b}(a b-b+1) & =\sum_{b=1}^{6}\left(b^{2}(b+1) / 2-b(b-1)\right)=\frac{1}{2} \sum_{b=1}^{6} b\left(b^{2}-b+2\right) \\
& =\frac{1}{2}(2+8+24+56+110+192)=196 \text { triplets. }
\end{aligned}
$$

Let $\mathcal{T}$ be the set of triplets given in the Table 1 . It contains 45 triplets (including $(6,6,8))$. At the end of the previous section we showed that each triplet in $\mathcal{T}$, except perhaps for $(6,6,8)$, is sum-feasible. So it remains to prove that none of the $196-45=151$ triplets in $\mathcal{A} \backslash \mathcal{T}$ is sum-feasible.

We first distinguish the set of 4 special triplets $\mathcal{S}$ defined in (12). The triplets of this set $(3,6,9),(4,6,8),(5,5,15)$ and $(6,6,10)$ are not sum-feasible by Theorem 33, Theorem 35, Corollary 37 and Theorem 38, respectively.

So we are left with the set $\mathcal{A} \backslash(\mathcal{T} \cup \mathcal{S})$ consisting of $151-4=$ 147 triplets. We next show that each triplet from the set $\mathcal{A} \backslash(\mathcal{T} \cup \mathcal{S})$ is not sum-feasible either by Proposition 2 or by Lemma 14. Those triplets in $\mathcal{A} \backslash(\mathcal{T} \cup \mathcal{S})$ that are not sum-feasible by Proposition 2 are given in Table 4 . In each case, the triplet contains a pair of coprime numbers but the third number is not their product. 
TABLE 4. Triplets that are not sum-feasible by Proposition 2.

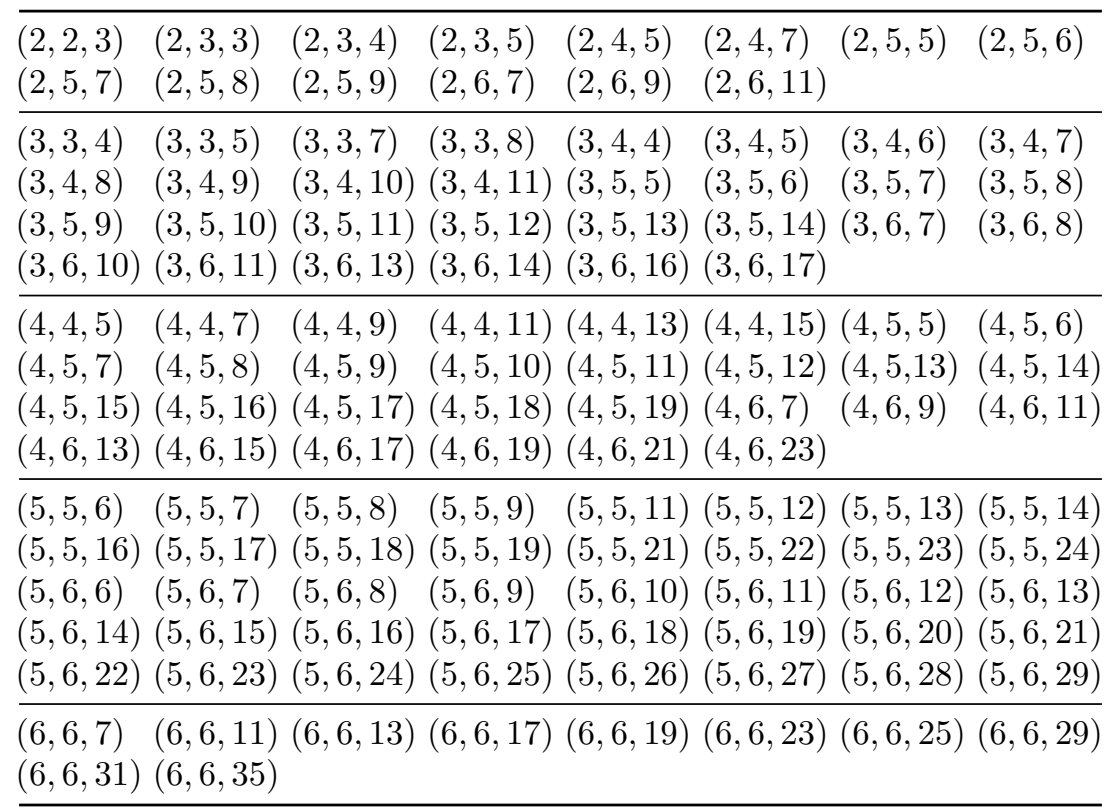

There are exactly 124 triplets in Table 4 . It remains to check the 'surviving' $147-124=23$ triplets that are in $\mathcal{A} \backslash(\mathcal{T} \cup \mathcal{S})$ but not in Table 4 . These are listed in Table 5 .

TABLE 5 . The 23 triplets that are not sum-feasible by Lemma 14 .

\begin{tabular}{l}
\hline$(2,4,6) \quad(2,6,8) \quad(2,6,10)$ \\
\hline$(3,6,15)$ \\
\hline$(4,4,10)(4,4,14)(4,6,10)(4,6,14)(4,6,16)(4,6,18)(4,6,20)(4,6,22)$ \\
\hline$(6,6,14)(6,6,16)(6,6,20)(6,6,21)(6,6,22)(6,6,26)(6,6,27)(6,6,28)$ \\
$(6,6,32)(6,6,33)(6,6,34)$ \\
\hline
\end{tabular}

One can easily check that each of those triplets is not sum-feasible, by Lemma 14 . 
Remark 39. Recall that triplet $(3,3,2)$ is product-feasible (see Section 1 ). The triplet $(2,2,4)$ satisfies the exponent triangle inequality with respect to any prime number. Hence the triplet $(6,6,8)=(3,3,2) \cdot(2,2,4)$ is product-feasible, by Proposition 28.

Acknowledgements. We thank A. Schinzel for providing several useful references. The first-named author acknowledges a postdoctoral fellowship funded by European Union Structural Funds project "Postdoctoral Fellowship Implementation in Lithuania".

\section{References}

[1] G. Baron, M. Drmota, and M. Skałba, Polynomial relations between polynomial roots, J. Algebra 177(3) (1995), 827-846. DOI: 10.1006/jabr.1995.1330.

[2] J. Browkin, B. Diviš, And A. Schinzel, Addition of sequences in general fields, Monatsh. Math. 82(4) (1976), 261-268. DOI: 10.1007/BF01540597.

[3] S. D. Cohen, A. Movahhedi, And A. Salinier, Double transitivity of Galois groups of trinomials, Acta Arith. 82(1) (1997), $1-15$.

[4] J. D. Dixon And B. Mortimer, "Permutation groups", Graduate Texts in Mathematics 163, Springer-Verlag, New York, 1996.

[5] M. Drmota and M. SkaŁba, On multiplicative and linear independence of polynomial roots, in: "Contributions to general algebra", 7 (Vienna, 1990), Hölder-Pichler-Tempsky, Vienna, 1991, pp. $127-135$.

[6] P. Drungilas, A. Dubickas, and F. LucA, On the degree of the compositum of two number fields, submitted.

[7] A. DubickAs, On the degree of a linear form in conjugates of an algebraic number, Illinois J. Math. 46(2) (2002), 571-585.

[8] A. Dubickas, Two exercises concerning the degree of the product of algebraic numbers, Publ. Inst. Math. (Beograd) (N.S.) 77(91) (2005), 67-70. DOI: 10.2298/PIM0591067D.

[9] A. Dubickas And C. J. SmYTh, Variations on the theme of Hilbert's Theorem 90, Glasg. Math. J. 44(3) (2002), 435-441. DOI: 10.1017/S0017089502030082.

[10] D. HilbeRT, "The theory of algebraic number fields", Translated from the German and with a preface by Iain T. Adamson. With an introduction by Franz Lemmermeyer and Norbert Schappacher, Springer-Verlag, Berlin, 1998. 
[11] I. M. Isaacs, Degrees of sums in a separable field extension, Proc. Amer. Math. Soc. 25 (1970), 638-641. DOI: 10.1090/S0002-99391970-0258803-3.

[12] J. JAHNEL, When is the (co)sine of a rational angle equal to a rational number? Preprint (2010), arXiv:1006.2938v1 [math.HO].

[13] C. Jensen, A. Ledet, And N. Yui, "Generic polynomials". Constructive aspects of the inverse Galois problem, Mathematical Sciences Research Institute Publications 45, Cambridge University Press, Cambridge, 2002.

[14] C. U. Jensen and N. Yui, Polynomials with $D_{p}$ as Galois group, J. Number Theory 15(3) (1982), 347-375. DOI: 10.1016/0022-314X (82) 90038-5.

[15] I. Kaplansky, "Fields and rings", The University of Chicago Press, Chicago, Ill.-London, 1969.

[16] S. Lang, "Algebra", Revised third edition, Graduate Texts in Mathematics 211, Springer-Verlag, New York, 2002.

[17] W. Ledermann, "Introduction to the theory of finite groups", $2 \mathrm{~d}$ ed., Oliver and Boyd, Edinburgh and London; Interscience Publishers, Inc., New York, 1953.

[18] A. Ledet, Dihedral extensions in characteristic 0, C. R. Math. Acad. Sci. Soc. R. Can. 21(2) (1999), 46-52.

[19] M. R. Murty And J. Esmonde, "Problems in algebraic number theory", Second edition, Graduate Texts in Mathematics 190, Springer-Verlag, New York, 2005.

[20] W. NARKIEWICZ, "Elementary and analytic theory of algebraic numbers", Third edition, Springer Monographs in Mathematics, Springer-Verlag, Berlin, 2004.

[21] A. R. Perlis, Roots appear in quanta, Amer. Math. Monthly 111(1) (2004), 61-63. DOI: $10.2307 / 4145020$.

[22] C. L. Siegel, "Über einige Anwendungen diophantischer Approximationen", Abh. Preuss. Akad. Wiss., 1929.

[23] C. J. Sмyтн, Conjugate algebraic numbers on conics, Acta Arith. 40(4) (1981/82), 333-346.

[24] C. J. Sмyтh, Additive and multiplicative relations connecting conjugate algebraic numbers, J. Number Theory 23(2) (1986), 243-254. DOI : 10.1016/0022-314X (86) 90094-6.

[25] J. L. Varona, Rational values of the arccosine function, Cent. Eur. J. Math. 4(2) (2006), 319-322 (electronic). DOI: 10.2478/s11533006-0011-z. 
Paulius Drungilas and Artūras Dubickas:

Department of Mathematics and Informatics

Vilnius University

Naugarduko 24

Vilnius LT-03225

Lithuania

E-mail address: pdrungilas@gmail.com

E-mail address: arturas.dubickas@mif.vu.lt

Chris Smyth:

School of Mathematics and

Maxwell Institute for Mathematical Science

University of Edinburgh

Edinburgh EH9 3JZ

Scotland, UK

E-mail address: c.smyth@ed.ac.uk

Primera versió rebuda el 14 de setembre de 2011, darrera versió rebuda el 23 de setembre de 2011. 